\title{
Clinopyroxene from basaltic rocks of the Erzgebirge-Krušné hory Mts. - implications for modelling of the magmatic plumbing system
}

\author{
Roman RÖNICK', Axel D. RENNO ${ }^{*}$ \\ ${ }^{1}$ Technische Universität Bergakademie Freiberg, Institute of Mineralogy, Brennhausgasse 14, D-09596 Freiberg, Germany; \\ axel.renno@mineral.tu-freiberg.de \\ * Corresponding author
}

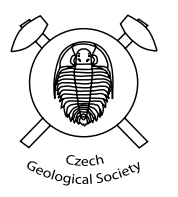

The Erzgebirge-Krušné hory area is part of the NW flank of the Eger-Ohře Rift. Within this rift-related area, basaltic rocks with low $\left(<3.5\right.$ wt. \%) and high contents $\left(>3.5\right.$ wt. \%) of $\mathrm{TiO}_{2}$ occur. The distribution of these basaltic rock types shows strong spatial dependency. Ti-rich basalts are concentrated in the western Erzgebirge-Krušné hory Mts. with a sharp change at longitude $13.3^{\circ} \mathrm{E}$. Clinopyroxene and olivine are the most abundant phenocrysts. The zoning and chemistry of the clinopyroxene act as a recorder of the different stages of the magmatic plumbing systems. Seven different types of clinopyroxene phenocrysts are defined with four distinct chemical types: Cr-rich clinopyroxene, green-core pyroxene, Mg-rich clinopyroxene and finely zoned Ti-rich clinopyroxene with sector zoning.

Chemical and petrographic analysis of individual zones within clinopyroxenes allows the qualitative description of distinct steps of the multistage, regionally diverse processes yielding to the formation of both Ti-rich basalts in the $\mathrm{W}$ as well as Ti-poor basalts in the E. Two different types of asthenospheric melts are suggested, with increased activities of $\mathrm{H}_{2} \mathrm{O}$ and potassium in the western Erzgebirge Mts. During ascent they incorporated xenoliths and xenocrysts of both the mantle (peridotite, olivine and Cr-rich clinopyroxene) and the crustal (granitic and gneissic rocks, quartz and feldspar) origin. Furthermore, green cores of clinopyroxene were introduced into the magmas. Fractionation processes took place within lithospheric magma chambers, having been accompanied by reaction of xenocrysts and xenoliths with the melt. The residence time in the magma chambers associated with the eastern and western parts of the Erzgebirge appears to have been totally different: short in the eastern and long in the western parts. Within the latter, no mantle-derived xenoliths, xenocrysts, or magmatic olivines are found. It is inferred that these xenoliths and crystals settled to the bottom of the magma chambers forming cumulates and therefore did not come to the surface. In contrast, the basaltic rocks of the East still contain mantle-derived xenoliths and xenocrysts as well as magmatic olivine. The size and amount of clinopyroxene phenocrysts in the West significantly exceed that of the East. Moreover, the crystallization of giant clinopyroxenes was restricted to the western region. Mixing of the magmas with Ti-rich melts, presumably derived from an eclogitic source, took place in crustal magma chambers. In any case, the proportion of Ti-rich melts in the West significantly exceeded that of the East.

Keywords: petrology, crystal chemistry, clinopyroxene, basalt, Erzgebirge-Krušné hory, green-core pyroxene Received: 6 April 2010; accepted: 20 September 2010; handling editor: V. Rapprich/R. Skála

The online version of this article (doi: 10.3190/jgeosci.077) contains supplementary electronic material.

\section{Introduction}

Despite the eye-catching geochemical character of ' $T i$ rich basalts' it is unclear if this feature is a vagary of nature or an important geochemical fingerprint able to discriminate between different source regions of basaltic rocks. Besides well known High-Ti basalts from the moon (Papike et al. 1976; Papike and Vaniman 1978), which have no terrestrial equivalent, High-Ti basalts are found in different geotectonic settings, such as Continental Flood Basalts (CFB), Large Igneous Provinces (LIP) (Gibson et al. 1995), or rift-like intraplate settings (Pik et al. 1998). The occurrence of such basalts is not restricted to lavas; they appear also in dike systems (Essawy and El-Metwally 1999; Katzir et al. 2006) and intrusions of gabbroic rocks (Jama Aden and Frizzo 1996). Their age spans from the Archaean to recent. The origin in each of the geotectonic environments remains controversial. The proposed explanations in LIPs range from a high degree of fractionation (Fodor 1987), low degree of partial melting (Dupuy et al. 1988), crustal contamination (Fodor 1987), to the influence of subcontinental lithosphere mobilized by subduction (Duncan 1987; Murphy 1988). It is assumed that the effect of mantle plumes is the main cause for the genesis of Ti-rich rocks in rift-like settings (Pik et al. 2006).

A qualitative approach to understanding petrological processes responsible for the genesis of Ti-rich basalts through the comprehensive study of the microstructure and mineral chemistry of clinopyroxene is demonstrated in the 
current paper. As a case study have been chosen Cenozoic basaltic rocks from the Eger-Ohře Rift (Erzgebirge-Krušné hory Mts, Bohemian Massif). Although we do not present a complete genetic model, this study does provide a basis for understanding the nature and genesis of the high-Ti basalts, both in this region and elsewhere.

\section{Geological setting}

According to Prodehl et al.(1995) the Eger-Ohře Rift, as part of the European Cenozoic Rift System (ECRS), is one of the major extensional structures in Central Europe. The structure itself is situated in Proterozoic to Paleozoic basement rocks of the Bohemian Massif (Malkovský 1987; Mlčoch and Konopásek 2010) and shows an elongation of roughly $300 \mathrm{~km}$ and a width up to $\sim 30 \mathrm{~km}$. The western part of the Eger Rift lies on a Variscan suture zone between two lithospheric blocks: the Saxothuringian and the Teplá-Barrandian terranes (Hrubcová et al. 2005; Babuška et al. 2007). The thermal thickness of the lithosphere at the level of the $1300{ }^{\circ} \mathrm{C}$ isotherm has been modelled to be $110-170 \mathrm{~km}$, whereas the mechanical thickness is only 65-90 km (Babuška and Plomerová 1992; Goes et al. 2000). Plomerová et al. (2007) with Babuška and Plomerová (this volume) found clear differences in the orientations of seismic anisotropy in the three major tectonic units (Saxothuringian, Moldanubian and Teplá-Barrandian), but no signs for the existence of plume-like structures in the mantle. The region shows evidence for repeated magmatic phases before the Tertiary but most of the Eger Rift lavas erupted between 30 and 15 Ma both within the rift and on its NW flank (Todt and Lippolt 1975; Pilot et al. 1984; Kaiser and Pilot 1986; Ulrych et al. 2002).

The NW-flank of the western part of the Eger Rift lies in the Erzgebirge-Krušné hory Mts. part of the Saxothuringian Zone of the Bohemian Massif, which is known to host volcanic rocks with distinct differences in the $\mathrm{TiO}_{2}$ contents (Pfeiffer 1978; Niese et al. 1995; Shrbený 1995; Ulrych et al. 2005; Haase and Renno 2008). Detailed studies of the mineral chemistry of clinopyroxene in the basaltic rocks of the Eger Rift are rare so far (Ulrych 1986; Ulrych et al. 1990; Rapprich 2005) and the current manuscript aims to bring new data in this respect.

\section{Sampling and methods}

The first step of sampling was the verification of all outcrops of basaltic rocks evinced in the $1: 25000$ geo-

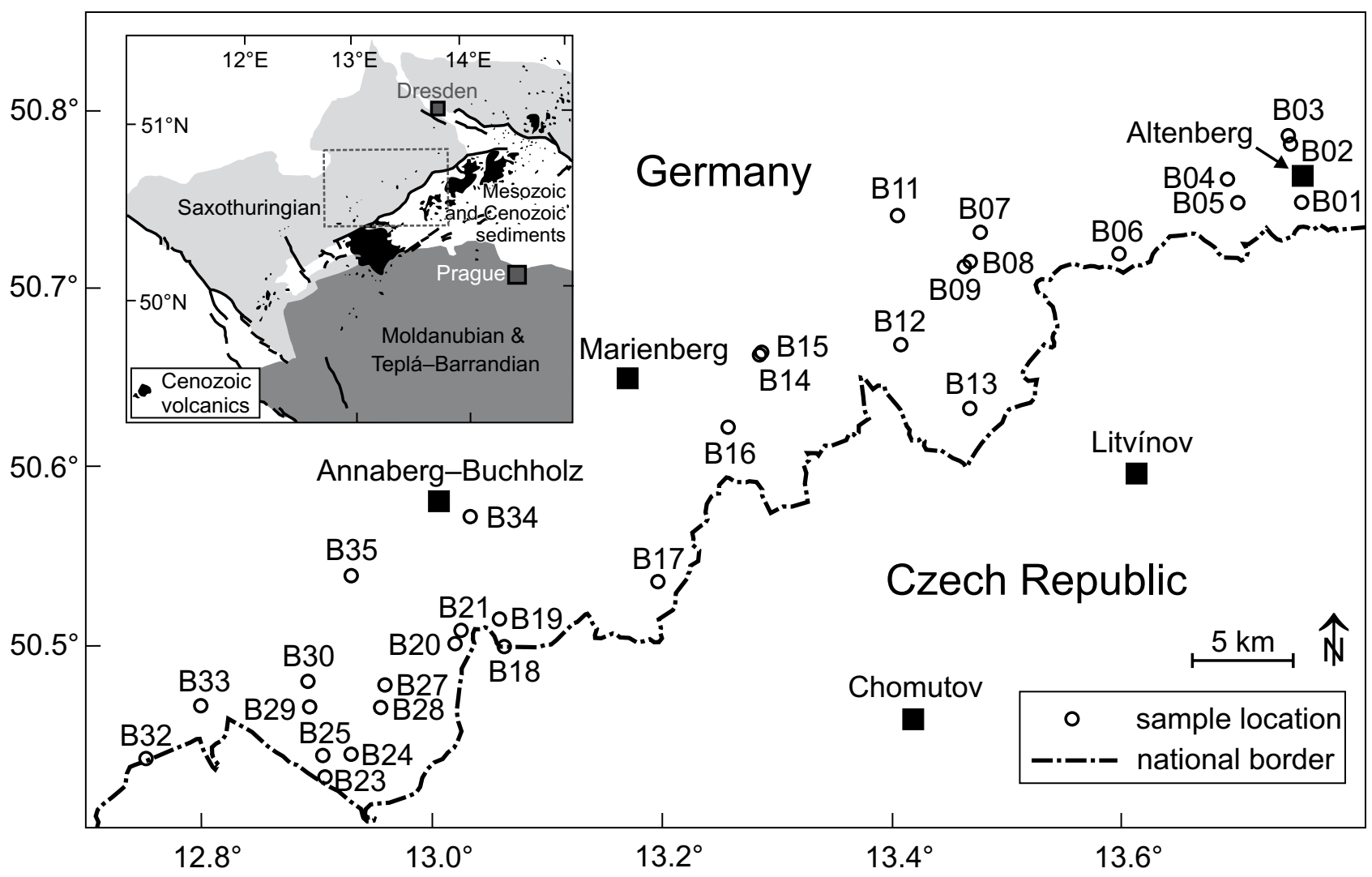

Fig. 1 Location of the samples. The locations, including GPS coordinates, are summarized in Tab. 1. 
logical maps of the German part of the Erzgebirge. The eventual sampling covered 27 localities (Tab. 1 and Fig 1). The samples were selected on the basis of the majorelement composition, i.e. only primitive rock types were chosen.

Fresh pieces were cut and washed in distilled water and an ultrasonic bath before crushing. The crushed rock sample was milled to powder in an agate ball mill. Whole-rock major-element analyses were measured with a Bruker "Tiger S8" X-ray fluorescence spectrometer at the Institut für Mineralogie, TU Bergakademie Freiberg using rock standards for calibration and quality control.

Mineral analyses were carried out using a JEOL JXA8900 electron microprobe at the Institut für Mineralogie, TU Bergakademie Freiberg. The analytical conditions for the minerals were an accelerating voltage of $15 \mathrm{keV}$, a beam current of $30 \mathrm{nA}$ and a focused beam. Counting times were $20-50 \mathrm{~s}$ for peak and $10-25 \mathrm{~s}$ for background positions. An accelerating voltage of $20 \mathrm{keV}$ and a beam current of $40 \mathrm{nA}$ were used for X-ray concentration maps and concentration profiles. Natural mineral standards and the ZAF matrix correction was employed.

All data were visualized using the GCDkit software package (Janoušek et al. 2006).

\section{Geochemistry}

Representative whole-rock analyses of the lavas are presented in Tab. 1. All the analyzed basaltic rocks represent truly alkali basaltic rocks, mostly basanites and mela- nephelinites (Supp. 1), according to the total alkali vs. $\mathrm{SiO}_{2}$ (TAS) classification scheme of Le Bas et al. (1986) and Le Maitre et al. (2005). We stuck to the proposals of Le Bas (1989) and Middlemost (1994) for further discrimination between basanite/tephrite and nephelinite/ melanephelinite (Fig. 2).

\subsection{Major elements and trace elements}

All samples form a continuous trend with decreasing $\mathrm{TiO}_{2}, \mathrm{FeO}^{\mathrm{T}}$, and $\mathrm{CaO}$ and increasing $\mathrm{MgO}$, and $\mathrm{Al}_{2} \mathrm{O}_{3}$ with increasing $\mathrm{SiO}_{2}$ (Fig. 3a-d and Supp. 2). With rising $\mathrm{FeO}^{\mathrm{T}} / \mathrm{MgO}$, ratio, often used as a differentiation index, $\mathrm{TiO}_{2}$, and $\mathrm{CaO}$ show a continuous increase, while $\mathrm{Cr}$, and Ni drop sharply (Fig. 3e and Supp. 2). The lavas show two separate positive trends in binary diagram of $\mathrm{FeO}^{\mathrm{T}}$ / $\mathrm{MgO}$ vs. $\mathrm{Al}_{2} \mathrm{O}_{3}$ (Fig. 3f). Some trace elements (e.g. Nb, $\mathrm{V}$, and $\mathrm{Zr}$ ) are positively correlated with $\mathrm{TiO}_{2}$ (Fig. $3 \mathrm{~g}$ and Supp. 2), whereas the ratio $\mathrm{CaO} / \mathrm{Al}_{2} \mathrm{O}_{3}$ shows a clear splitting at c. 3.5 wt. $\% \mathrm{TiO}_{2}$ (Fig. 3h).

\subsection{Regional variation}

A striking feature is the regional dichotomy in wholerock compositions of basalts from the eastern and western Erzgebirge. At a longitude of $13.3^{\circ} \mathrm{E}$ we observed an abrupt change (Supp. 3), best exemplified by $\mathrm{TiO}_{2}$ (Fig. 4). Despite a small overlap, $\mathrm{TiO}_{2}$ (threshold value 3.5 wt. \%) and $\mathrm{CaO}$ (threshold value 13.5 wt. \%) drop eastwards, while $\mathrm{Al}_{2} \mathrm{O}_{3}$ (threshold value 12 wt. \%),
Fig. 2 Total alkali vs. $\mathrm{SiO}_{2}$ (TAS) diagram for the analysed basaltic rocks and some additional samples from the same area (Haase and Renno 2008).
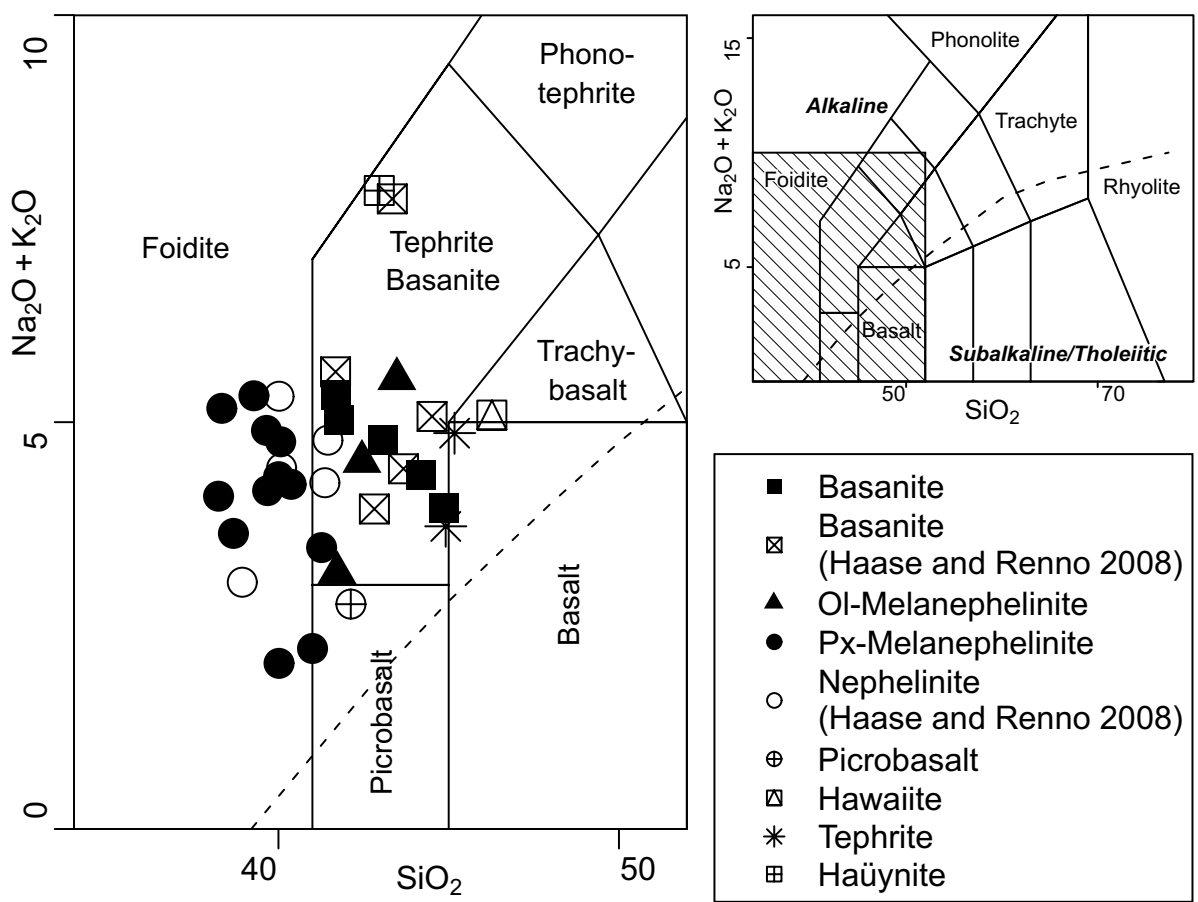

\begin{tabular}{|c|c|}
\hline 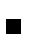 & Basanite \\
\hline$\nabla$ & $\begin{array}{l}\text { Basanite } \\
\text { (Haase and Renno 2008) }\end{array}$ \\
\hline$\Delta$ & Ol-Melanephelinite \\
\hline & Px-Melanephelinite \\
\hline 0 & $\begin{array}{l}\text { Nephelinite } \\
\text { (Haase and Renno 2008) }\end{array}$ \\
\hline$\oplus$ & Picrobasalt \\
\hline$\Delta$ & Hawaiite \\
\hline * & Tephrite \\
\hline$\boxplus$ & Haüynite \\
\hline
\end{tabular}


Tab. 1 Whole-rock major- and trace-element compositions for the studied basaltic rocks from the eastern and western Erzgebirge (Germany)

\begin{tabular}{|c|c|c|c|c|c|c|c|}
\hline Sample & B01 & B02a & B03a & B05 & B06 & B07 & B08 \\
\hline Locality & NNW Zinnwald & SE Hirschsprung & Hirschsprung & $\begin{array}{l}\text { road Altenberg- } \\
\text { Rehefeld }\end{array}$ & $\begin{array}{c}\text { Steinkuppe } \\
\text { near Holzhau }\end{array}$ & $\begin{array}{c}\text { Ziegenberg } \\
\text { near Claußnitz }\end{array}$ & "Kreuztanne" \\
\hline Latitude $^{\circ} \mathbf{N}$ & 50.749 & 50.782 & 50.786 & 50.749 & 50.720 & 50.730 & 50.715 \\
\hline Longitude ${ }^{\circ} \mathbf{E}$ & 13.76 & 13.75 & 13.75 & 13.71 & 13.60 & 13.47 & 13.47 \\
\hline Rock type & basanite & basanite & basanite & Ol-Mn & Ol-Mn & Ol-Mn & hawaiite \\
\hline $\mathrm{SiO}_{2}$ & 42.29 & 40.97 & 40.71 & 40.27 & 42.34 & 41.75 & 45.13 \\
\hline $\mathrm{TiO}_{2}$ & 2.72 & 3.71 & 3.59 & 3.37 & 3.14 & 3.07 & 3.20 \\
\hline $\mathrm{Al}_{2} \mathrm{O}_{3}$ & 12.68 & 13.72 & 13.11 & 12.66 & 13.44 & 13.26 & 13.63 \\
\hline $\mathrm{Fe}_{2} \mathrm{O}_{3} \mathrm{t}$ & 12.39 & 13.36 & 13.27 & 13.91 & 12.37 & 12.92 & 12.25 \\
\hline MgO & 12.17 & 9.93 & 10.84 & 9.99 & 9.18 & 10.57 & 8.80 \\
\hline MnO & 0.19 & 0.19 & 0.19 & 0.23 & 0.20 & 0.21 & 0.22 \\
\hline $\mathrm{CaO}$ & 11.67 & 11.75 & 11.46 & 13.39 & 11.73 & 12.74 & 9.88 \\
\hline $\mathrm{Na}_{2} \mathrm{O}$ & 3.32 & 3.75 & 3.63 & 2.55 & 4.25 & 3.59 & 3.66 \\
\hline $\mathrm{K}_{2} \mathrm{O}$ & 1.37 & 1.49 & 1.27 & 0.49 & 1.13 & 0.85 & 1.30 \\
\hline $\mathbf{P}_{2} \mathbf{O}_{5}$ & 0.60 & 0.73 & 0.71 & 1.06 & 0.83 & 0.68 & 0.69 \\
\hline L.o.I. & 0.23 & 0.40 & 0.35 & 0.88 & 0.50 & 0.40 & 0.46 \\
\hline Total & 99.63 & 100.01 & 99.13 & 98.79 & 99.11 & 100.03 & 99.22 \\
\hline B & 76 & 128 & 134 & 100 & 112 & 97 & 112 \\
\hline $\mathbf{F}$ & 491 & 897 & 776 & 252 & 898 & 528 & 869 \\
\hline $\mathbf{S}$ & 190 & 270 & 230 & 190 & 260 & 240 & 470 \\
\hline Cl & 179 & 250 & 218 & bd & 212 & bd & bd \\
\hline $\mathbf{V}$ & 223 & 268 & 261 & 252 & 271 & 254 & 211 \\
\hline $\mathrm{Cr}$ & 361 & 136 & 188 & 157 & 189 & 290 & 102 \\
\hline Co & 51 & 46 & 48 & 43 & 41 & 48 & 40 \\
\hline $\mathbf{N i}$ & 209 & 108 & 152 & 130 & 110 & 138 & 90 \\
\hline $\mathrm{Cu}$ & 48 & 61 & 53 & 31 & 38 & 43 & 35 \\
\hline $\mathrm{Zn}$ & 97 & 97 & 97 & 97 & 97 & 97 & 97 \\
\hline Ga & 17 & 18 & 17 & 17 & 18 & 17 & 18 \\
\hline As & 2 & 3 & 3 & 5 & 8 & 4 & 19 \\
\hline $\mathrm{Br}$ & 4 & 4 & 4 & 2 & 4 & 3 & 3 \\
\hline $\mathbf{R b}$ & 32 & 53 & 48 & 49 & 39 & 45 & 49 \\
\hline $\mathrm{Sr}$ & 618 & 710 & 688 & 1344 & 782 & 701 & 707 \\
\hline $\mathbf{Y}$ & 20 & 21 & 20 & 22 & 23 & 22 & 22 \\
\hline $\mathrm{Zr}$ & 206 & 236 & 226 & 270 & 264 & 226 & 243 \\
\hline Nb & 60 & 68 & 68 & 58 & 71 & 57 & 55 \\
\hline Mo & bd & 1 & 4 & 2 & 4 & 2 & 4 \\
\hline Ag & bd & bd & bd & bd & bd & bd & bd \\
\hline Cd & bd & bd & bd & bd & 1 & bd & 1 \\
\hline Sn & 5 & 7 & 5 & 9 & 4 & 6 & 4 \\
\hline Cs & 0.6 & 2.4 & 1.2 & bd & 2.2 & 5.7 & 39.8 \\
\hline Ba & 434 & 499 & 468 & 707 & 632 & 542 & 668 \\
\hline La & 38 & 52 & 55 & 83 & 76 & 49 & 55 \\
\hline $\mathrm{Ce}$ & 86 & 90 & 87 & 133 & 125 & 99 & 93 \\
\hline $\mathbf{P b}$ & 4 & 5 & 2 & 7 & bd & 4 & bd \\
\hline $\mathbf{U}$ & 11.2 & 11.4 & 11.5 & 15.6 & 11.2 & 11.0 & 10.2 \\
\hline
\end{tabular}

$\mathrm{bd}=$ below detection limit; $\mathrm{Mn}=$ melanephelinite 
Tab. 1 Continued

\begin{tabular}{|c|c|c|c|c|c|c|c|}
\hline Sample & B11b & B12b & B13 & B14a & B15 & B16 & B17 \\
\hline Locality & $\begin{array}{c}\text { Galgenberg } \\
\text { (road way } \\
\text { Voigtsdorf-Sayda) }\end{array}$ & $\begin{array}{r}\text { Schafferholz } \\
\text { (Heidersdorf) }\end{array}$ & $\begin{array}{l}\text { Ahornberg } \\
\text { (Seiffen) }\end{array}$ & $\begin{array}{c}\text { SW } \\
\text { Blumenau }\end{array}$ & SW Blumenau & $\begin{array}{l}\text { Rabenberg } \\
\text { (SE Zöblitz) }\end{array}$ & $\begin{array}{c}\text { Hirtstein } \\
\text { (N Satzung) }\end{array}$ \\
\hline Latitude $^{\circ} \mathbf{N}$ & 50.740 & 50.668 & 50.632 & 50.662 & 50.663 & 50.622 & 50.536 \\
\hline Longitude $^{\circ} \mathbf{E}$ & 13.40 & 13.41 & 13.47 & 13.28 & 13.28 & 13.25 & 13.19 \\
\hline Rock type & basanite & basalt & basanite & Cpx-Mn & picrobasalt & Cpx-Mn & Cpx-Mn \\
\hline $\mathrm{SiO}_{2}$ & 43.74 & 47.24 & 43.63 & 39.63 & 41.23 & 39.23 & 39.13 \\
\hline $\mathrm{TiO}_{2}$ & 2.98 & 2.72 & 2.36 & 3.88 & 3.83 & 4.32 & 4.04 \\
\hline $\mathrm{Al}_{2} \mathrm{O}_{3}$ & 12.40 & 13.48 & 11.09 & 10.16 & 10.30 & 11.17 & 10.42 \\
\hline $\mathrm{Fe}_{2} \mathrm{O}_{3} \mathrm{t}$ & 11.75 & 11.12 & 12.01 & 14.31 & 14.22 & 14.94 & 14.47 \\
\hline MgO & 10.53 & 8.27 & 16.01 & 9.07 & 9.36 & 8.26 & 8.85 \\
\hline MnO & 0.18 & 0.18 & 0.18 & 0.21 & 0.20 & 0.22 & 0.22 \\
\hline $\mathrm{CaO}$ & 12.29 & 11.31 & 9.66 & 17.97 & 16.75 & 15.54 & 16.45 \\
\hline $\mathrm{Na}_{2} \mathrm{O}$ & 2.95 & 3.12 & 3.07 & 1.63 & 1.91 & 2.95 & 2.83 \\
\hline $\mathbf{K}_{2} \mathbf{O}$ & 0.90 & 0.99 & 1.23 & 0.51 & 0.80 & 1.16 & 1.82 \\
\hline $\mathbf{P}_{2} \mathbf{O}_{5}$ & 0.93 & 0.50 & 0.70 & 0.72 & 0.70 & 0.86 & 0.90 \\
\hline L.o.I. & 0.51 & 0.76 & 0.11 & 0.94 & 0.55 & 0.36 & 0.56 \\
\hline Total & 99.17 & 99.68 & 100.06 & 99.03 & 99.84 & 99.01 & 99.67 \\
\hline B & 115 & 109 & 88 & 126 & 118 & 146 & 146 \\
\hline $\mathbf{F}$ & 505 & 375 & bd & 1344 & 1245 & 692 & 1385 \\
\hline $\mathbf{S}$ & 300 & 240 & 110 & 910 & 580 & 300 & 320 \\
\hline Cl & bd & bd & 85 & 396 & 170 & bd & bd \\
\hline $\mathbf{V}$ & 242 & 237 & 183 & 383 & 371 & 416 & 393 \\
\hline $\mathrm{Cr}$ & 299 & 272 & 549 & 185 & 193 & 100 & 100 \\
\hline Co & 43 & 41 & 60 & 46 & 49 & 46 & 49 \\
\hline $\mathbf{N i}$ & 136 & 130 & 445 & 56 & 59 & 38 & 50 \\
\hline $\mathrm{Cu}$ & 50 & 43 & 35 & 197 & 196 & 244 & 283 \\
\hline $\mathbf{Z n}$ & 97 & 97 & 97 & 97 & 97 & 97 & 97 \\
\hline Ga & 17 & 17 & 17 & 17 & 18 & 19 & 18 \\
\hline As & 3 & 7 & 6 & 2 & 5 & 1 & 5 \\
\hline $\mathrm{Br}$ & 3 & 3 & 4 & 4 & 3 & 3 & 3 \\
\hline $\mathbf{R b}$ & 38 & 207 & 32 & 58 & 45 & 49 & 43 \\
\hline $\mathrm{Sr}$ & 768 & 548 & 765 & 701 & 729 & 790 & 804 \\
\hline $\mathbf{Y}$ & 23 & 28 & 18 & 18 & 19 & 23 & 21 \\
\hline $\mathbf{Z r}$ & 237 & 198 & 221 & 231 & 237 & 280 & 305 \\
\hline Nb & 67 & 48 & 69 & 77 & 74 & 96 & 95 \\
\hline Mo & 3 & 1 & 1 & 2 & bd & bd & bd \\
\hline Ag & bd & bd & bd & bd & bd & bd & bd \\
\hline Cd & bd & bd & bd & bd & bd & bd & bd \\
\hline Sn & 4 & 6 & 6 & 6 & 6 & 3 & 3 \\
\hline Cs & 1.5 & 5.5 & 0.7 & 1.7 & 2.2 & bd & bd \\
\hline $\mathbf{B a}$ & 602 & 867 & 448 & 585 & 898 & 978 & 531 \\
\hline La & 70 & 42 & 55 & 63 & 64 & 84 & 83 \\
\hline $\mathrm{Ce}$ & 124 & 83 & 125 & 113 & 117 & 148 & 135 \\
\hline $\mathbf{P b}$ & 4 & 6 & 5 & 6 & 5 & 3 & 6 \\
\hline $\mathbf{U}$ & 11.3 & 6.0 & 12.5 & 10.4 & 8.1 & 7.9 & 11.7 \\
\hline
\end{tabular}

bd = below detection limit; $\mathrm{Mn}=$ melanephelinite 
Tab. 1 Continued

\begin{tabular}{|c|c|c|c|c|c|c|c|}
\hline Sample & B18c & B19 & B21 & B24 & B25 & B27a & B30b \\
\hline Locality & $\begin{array}{l}\text { SW Jöhstadt } \\
\text { (state frontier) }\end{array}$ & $\begin{array}{l}\text { Klöfs-Berg } \\
\text { (W Jöhstadt) }\end{array}$ & $\begin{array}{c}\text { Bärenstein } \\
\text { (W Bärenstein) }\end{array}$ & $\begin{array}{l}\text { Wurzelbergstraße } \\
\text { (Oberwiesenthal) }\end{array}$ & $\begin{array}{c}\text { Einsberg } \\
\text { (NE Tellerhäuser) }\end{array}$ & $\begin{array}{l}\text { Morgenberg } \\
\text { (W Neudorf) }\end{array}$ & $\begin{array}{c}\text { Eisensteinberg } \\
\text { (W Neudorf) }\end{array}$ \\
\hline Latitude ${ }^{\circ} \mathbf{N}$ & 50.501 & 50.516 & 50.508 & 50.442 & 50.439 & 50.479 & 50.481 \\
\hline Longitude ${ }^{\circ} \mathbf{E}$ & 13.06 & 13.05 & 13.02 & 12.93 & 12.90 & 12.96 & 12.89 \\
\hline Rock type & Ol-Cpx-leucitite & Cpx-Mn & Cpx-Mn & tephrite & Cpx-Mn & haüynite & Cpx-Mn \\
\hline $\mathrm{SiO}_{2}$ & 40.79 & 38.91 & 38.18 & 43.74 & 40.31 & 41.56 & 38.76 \\
\hline $\mathrm{TiO}_{2}$ & 3.95 & 4.37 & 5.02 & 3.75 & 4.68 & 4.12 & 4.75 \\
\hline $\mathrm{Al}_{2} \mathrm{O}_{3}$ & 10.71 & 10.38 & 11.04 & 12.99 & 11.55 & 13.79 & 11.24 \\
\hline $\mathrm{Fe}_{2} \mathrm{O}_{3} \mathrm{t}$ & 13.89 & 16.09 & 16.23 & 12.92 & 15.01 & 12.65 & 14.74 \\
\hline MgO & 10.11 & 8.31 & 7.05 & 6.74 & 7.26 & 4.32 & 8.25 \\
\hline MnO & 0.18 & 0.21 & 0.27 & 0.20 & 0.22 & 0.28 & 0.23 \\
\hline $\mathrm{CaO}$ & 14.81 & 16.64 & 14.61 & 12.42 & 15.91 & 12.36 & 15.58 \\
\hline $\mathrm{Na}_{2} \mathrm{O}$ & 2.27 & 2.79 & 3.93 & 3.59 & 2.60 & 4.20 & 3.30 \\
\hline $\mathbf{K}_{2} \mathbf{O}$ & 2.14 & 1.29 & 1.25 & 1.12 & 0.78 & 3.39 & 1.49 \\
\hline $\mathbf{P}_{2} \mathbf{O}_{5}$ & 0.62 & 0.69 & 1.26 & 0.65 & 0.87 & 1.33 & 0.88 \\
\hline L.o.I. & 0.25 & 0.27 & 0.44 & 0.67 & 0.63 & 0.52 & 0.55 \\
\hline Total & 99.72 & 99.95 & 99.28 & 98.79 & 99.83 & 98.52 & 99.76 \\
\hline B & 112 & 146 & 160 & 126 & 162 & 180 & 169 \\
\hline $\mathbf{F}$ & 560 & 1370 & 1643 & 1376 & 1443 & 1915 & 2155 \\
\hline $\mathbf{S}$ & 330 & 410 & 270 & 1020 & 390 & 2100 & 600 \\
\hline Cl & bd & bd & bd & bd & bd & 1517 & 200 \\
\hline $\mathbf{V}$ & 369 & 454 & 408 & 324 & 410 & 352 & 393 \\
\hline $\mathrm{Cr}$ & 270 & 26 & 12 & 62 & 37 & 10 & 88 \\
\hline Co & 48 & 53 & 45 & 44 & 48 & 27 & 45 \\
\hline $\mathbf{N i}$ & 80 & 38 & 28 & 53 & 31 & 11 & 53 \\
\hline $\mathrm{Cu}$ & 247 & 414 & 82 & 81 & 262 & 31 & 178 \\
\hline $\mathbf{Z n}$ & 97 & 97 & 112 & 97 & 99 & 131 & 97 \\
\hline Ga & 17 & 20 & 21 & 19 & 20 & 25 & 19 \\
\hline As & 4 & 5 & 2 & 7 & 3 & 7 & 4 \\
\hline $\mathrm{Br}$ & 3 & 4 & 3 & 3 & 3 & 7 & 4 \\
\hline $\mathbf{R b}$ & 70 & 36 & 40 & 46 & 82 & 77 & 37 \\
\hline $\mathrm{Sr}$ & 456 & 833 & 899 & 788 & 731 & 1498 & 829 \\
\hline $\mathbf{Y}$ & 20 & 19 & 27 & 23 & 22 & 38 & 22 \\
\hline $\mathbf{Z r}$ & 220 & 262 & 377 & 247 & 306 & 523 & 293 \\
\hline $\mathbf{N b}$ & 76 & 89 & 107 & 76 & 89 & 157 & 94 \\
\hline Mo & bd & bd & $\mathrm{bd}$ & 9 & bd & 3 & bd \\
\hline Ag & bd & bd & bd & $\mathrm{bd}$ & bd & bd & bd \\
\hline Cd & bd & bd & 1 & bd & 1 & bd & bd \\
\hline Sn & 7 & 4 & 6 & 6 & 8 & 8 & 5 \\
\hline Cs & bd & 0.6 & bd & bd & bd & bd & bd \\
\hline $\mathbf{B a}$ & 1024 & 697 & 511 & 664 & 439 & 954 & 621 \\
\hline La & 62 & 76 & 108 & 74 & 72 & 148 & 86 \\
\hline $\mathrm{Ce}$ & 123 & 117 & 169 & 121 & 117 & 260 & 142 \\
\hline $\mathbf{P b}$ & 4 & 6 & bd & 7 & bd & 16 & 5 \\
\hline $\mathbf{U}$ & 4.4 & 10.5 & 12.9 & 10.9 & 11.6 & 14.4 & 11.3 \\
\hline
\end{tabular}

bd $=$ below detection limit; $\mathrm{Mn}=$ melanephelinite 
Tab. 1 Continued

\begin{tabular}{|c|c|c|c|c|c|c|}
\hline Sample & B30c & B32 & B33a & B33b & B34 & B35 \\
\hline Locality & $\begin{array}{l}\text { Eisensteinberg } \\
\text { (W Neudorf) }\end{array}$ & $\begin{array}{c}\text { Glücksburg Berg } \\
\text { (E Johanngeorgenstadt }\end{array}$ & $\begin{array}{l}\text { Vordere Kohlung } \\
\text { (S Rittersgrün) }\end{array}$ & $\begin{array}{l}\text { Vordere Kohlung } \\
\text { (S Rittersgrün) }\end{array}$ & $\begin{array}{c}\text { Pöhlberg } \\
\text { (E Annaberg-Buchholz) }\end{array}$ & $\begin{array}{c}\text { Scheibenberg } \\
\text { (E Scheibenberg) }\end{array}$ \\
\hline Latitude $^{\circ} \mathbf{N}$ & 50.481 & 50.436 & 50.467 & 50.467 & 50.574 & 50.540 \\
\hline Longitude ${ }^{\circ} \mathbf{E}$ & 12.89 & 12.75 & 12.80 & 12.80 & 13.03 & 12.92 \\
\hline Rock type & tephrite & Cpx-Mn & Cpx-Mn & Cpx-Mn & Cpx-Mn & Cpx-Mn \\
\hline $\mathrm{SiO}_{2}$ & 43.63 & 38.94 & 37.10 & 39.17 & 37.20 & 37.79 \\
\hline $\mathrm{TiO}_{2}$ & 4.18 & 4.81 & 4.97 & 4.39 & 5.03 & 5.12 \\
\hline $\mathrm{Al}_{2} \mathrm{O}_{3}$ & 11.02 & 10.28 & 11.42 & 10.92 & 11.33 & 11.03 \\
\hline $\mathrm{Fe}_{2} \mathrm{O}_{3} \mathrm{t}$ & 12.55 & 15.29 & 15.30 & 15.21 & 15.74 & 16.19 \\
\hline MgO & 7.98 & 10.46 & 6.35 & 8.25 & 6.37 & 7.92 \\
\hline MnO & 0.19 & 0.21 & 0.25 & 0.21 & 0.26 & 0.22 \\
\hline $\mathrm{CaO}$ & 14.48 & 16.13 & 17.86 & 16.24 & 16.11 & 16.51 \\
\hline $\mathrm{Na}_{2} \mathrm{O}$ & 2.35 & 1.50 & 2.99 & 2.84 & 3.65 & 2.57 \\
\hline $\mathrm{K}_{2} \mathrm{O}$ & 1.27 & 0.49 & 0.98 & 1.40 & 1.36 & 0.98 \\
\hline $\mathbf{P}_{2} \mathbf{O}_{5}$ & 0.76 & 0.75 & 1.33 & 0.81 & 1.56 & 1.00 \\
\hline L.o.I. & 0.77 & 1.01 & 0.45 & 0.34 & 0.42 & 0.58 \\
\hline Total & 99.17 & 99.85 & 99.01 & 99.77 & 99.03 & 99.90 \\
\hline B & 155 & 163 & 176 & 155 & 164 & 151 \\
\hline $\mathbf{F}$ & 1734 & 1734 & 1671 & 1446 & 2466 & 2034 \\
\hline $\mathbf{S}$ & 1940 & 530 & 960 & 470 & 1050 & 820 \\
\hline Cl & bd & 115 & bd & bd & 425 & $\mathrm{bd}$ \\
\hline $\mathbf{V}$ & 350 & 389 & 437 & 422 & 464 & 475 \\
\hline $\mathrm{Cr}$ & 116 & 193 & 10 & 74 & 17 & 49 \\
\hline Co & 42 & 52 & 44 & 50 & 52 & 54 \\
\hline $\mathbf{N i}$ & 60 & 82 & 12 & 39 & 21 & 39 \\
\hline $\mathrm{Cu}$ & 148 & 213 & 295 & 330 & 186 & 287 \\
\hline $\mathbf{Z n}$ & 97 & 97 & 104 & 97 & 122 & 106 \\
\hline Ga & 17 & 17 & 20 & 19 & 24 & 22 \\
\hline As & 5 & 5 & 4 & 3 & 5 & 5 \\
\hline $\mathrm{Br}$ & 3 & 4 & 3 & 3 & 5 & 4 \\
\hline $\mathbf{R b}$ & 77 & 53 & 50 & 44 & 56 & 47 \\
\hline $\mathrm{Sr}$ & 1009 & 667 & 998 & 857 & 822 & 807 \\
\hline $\mathbf{Y}$ & 22 & 19 & 25 & 20 & 36 & 31 \\
\hline $\mathbf{Z r}$ & 253 & 258 & 352 & 270 & 426 & 345 \\
\hline $\mathrm{Nb}$ & 89 & 68 & 90 & 83 & 114 & 106 \\
\hline Mo & 4 & bd & 1 & bd & bd & 2 \\
\hline Ag & bd & bd & bd & bd & 2.3 & bd \\
\hline Cd & bd & bd & bd & 1 & bd & bd \\
\hline Sn & 8 & 7 & 6 & 7 & 7 & 6 \\
\hline Cs & 0.3 & bd & bd & bd & 2.8 & bd \\
\hline $\mathbf{B a}$ & 662 & 540 & 745 & 903 & 302 & 610 \\
\hline La & 79 & 74 & 95 & 74 & 98 & 89 \\
\hline $\mathrm{Ce}$ & 135 & 131 & 173 & 136 & 179 & 144 \\
\hline $\mathbf{P b}$ & 6 & 5 & 8 & 1 & 12 & 10 \\
\hline $\mathbf{U}$ & 12.6 & 10.4 & 11.6 & 9.1 & 12.9 & 10.1 \\
\hline
\end{tabular}

$\mathrm{bd}=$ below detection limit; $\mathrm{Mn}=$ melanephelinite 

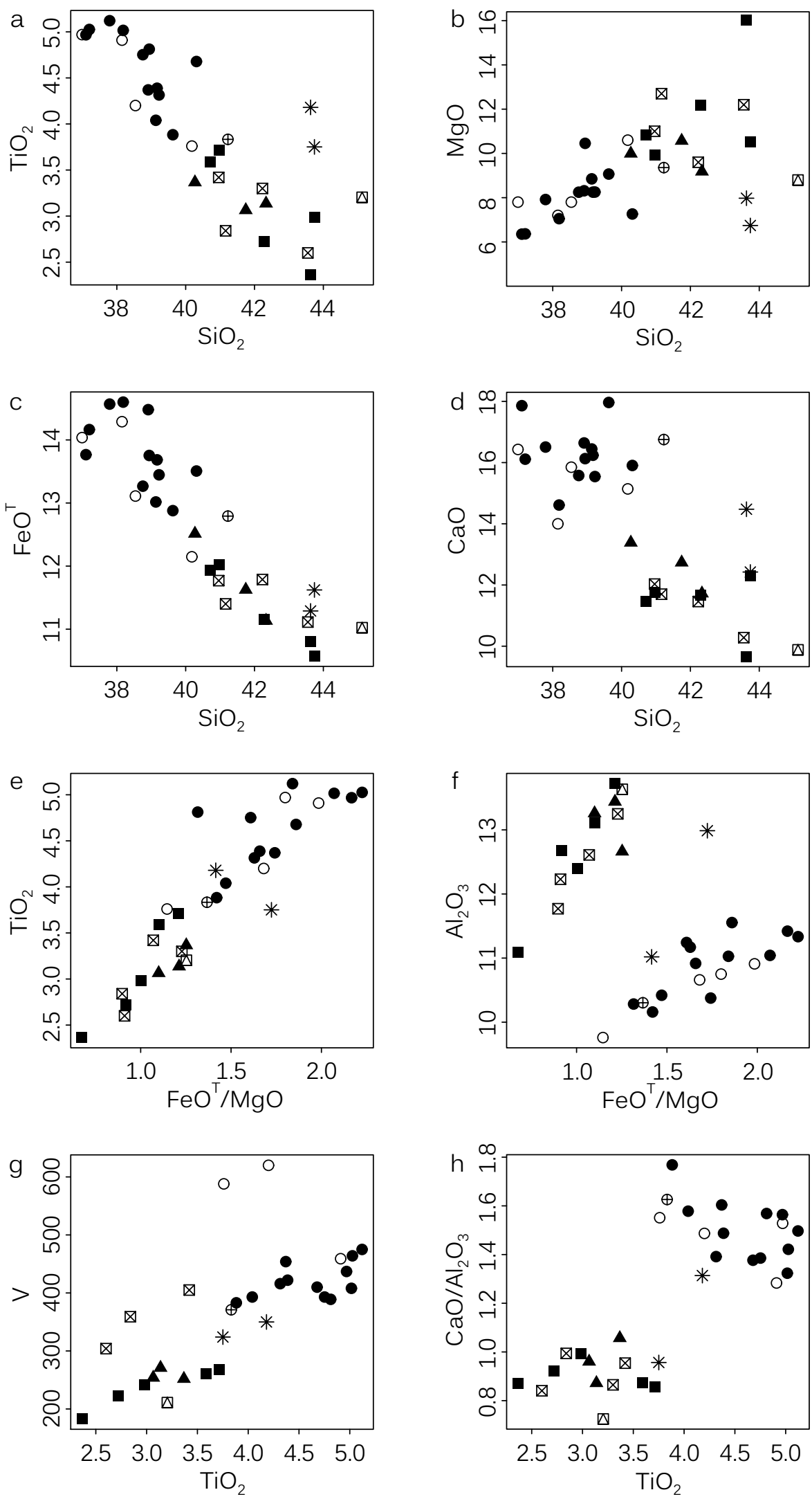

Fig. 3 Selected variation diagrams for the Erzgebirge basaltic rocks. Symbols and additional data sources as in Fig. 2. a $-\mathrm{SiO}_{2}$ vs. $\mathrm{TiO}_{2} ; \mathbf{b}-\mathrm{SiO}_{2}$ vs. $\mathrm{MgO} ; \mathbf{c}-\mathrm{SiO}_{2}$ vs. $\mathrm{FeO}^{\mathrm{T}} ; \mathbf{d}-\mathrm{SiO}_{2}$ vs. $\mathrm{CaO} ; \mathbf{e}-\mathrm{FeO}^{\mathrm{T}} /$ $\mathrm{MgO}$ vs. $\mathrm{TiO}_{2} ; \mathbf{f}-\mathrm{FeO}^{\mathrm{T}} / \mathrm{MgO}$ vs. $\mathrm{Al}_{2} \mathrm{O}_{3} ; \mathbf{g}-\mathrm{TiO}_{2}$ vs. $\mathrm{V} ; \mathbf{h}-\mathrm{TiO}_{2}$ vs. $\mathrm{CaO} / \mathrm{Al}_{2} \mathrm{O}_{3}$. and mg\# (threshold value 58) increase. Such variations are known from other parts of the ECRS like the Vogelsberg (Bogaard and Wörner 2003) and the Rhön (Jung and Hoernes 2000).

\section{Petrography}

The petrography of all samples was described in detail by Rönick (2010). The main features are compiled in Supp. 1.
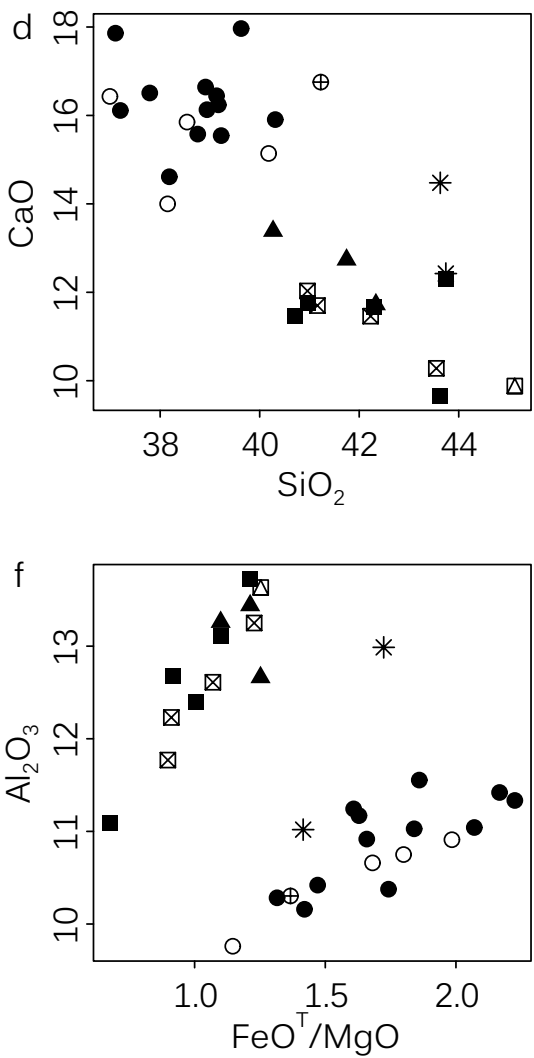

\subsection{Groundmass}

All rocks show a porphyritic microstructure with a fine-grained groundmass. This groundmass consists of small, elongate clinopyroxene and isometric opaque minerals. Some lavas show flow patterns or schlieren-like inhomogeneities. Additionally minerals of the sodalite-group, nepheline, perovskite, and analcime occur, predominantly in the rocks from the western part of the Erzgebirge. Lath-shaped plagioclase is typical of the lavas from the eastern Erzgebirge. Apatite is a common component of the groundmass, but can in some cases reach the size of a phenocryst. Tiny flakes of biotite (c. $0.02 \mathrm{~mm}$ ) are found in many samples from both the eastern and the western Erzgebirge.

\subsection{Phenocrysts}

The most common phenocrysts are clinopyroxene and olivine; amphibole and biotite are of subordinate importance.

\subsubsection{Olivine}

Olivine is found almost exclusively in lavas from the eastern Erzgebirge and forms the main constituent of the phenocrysts in these rocks. Most of the olivine 


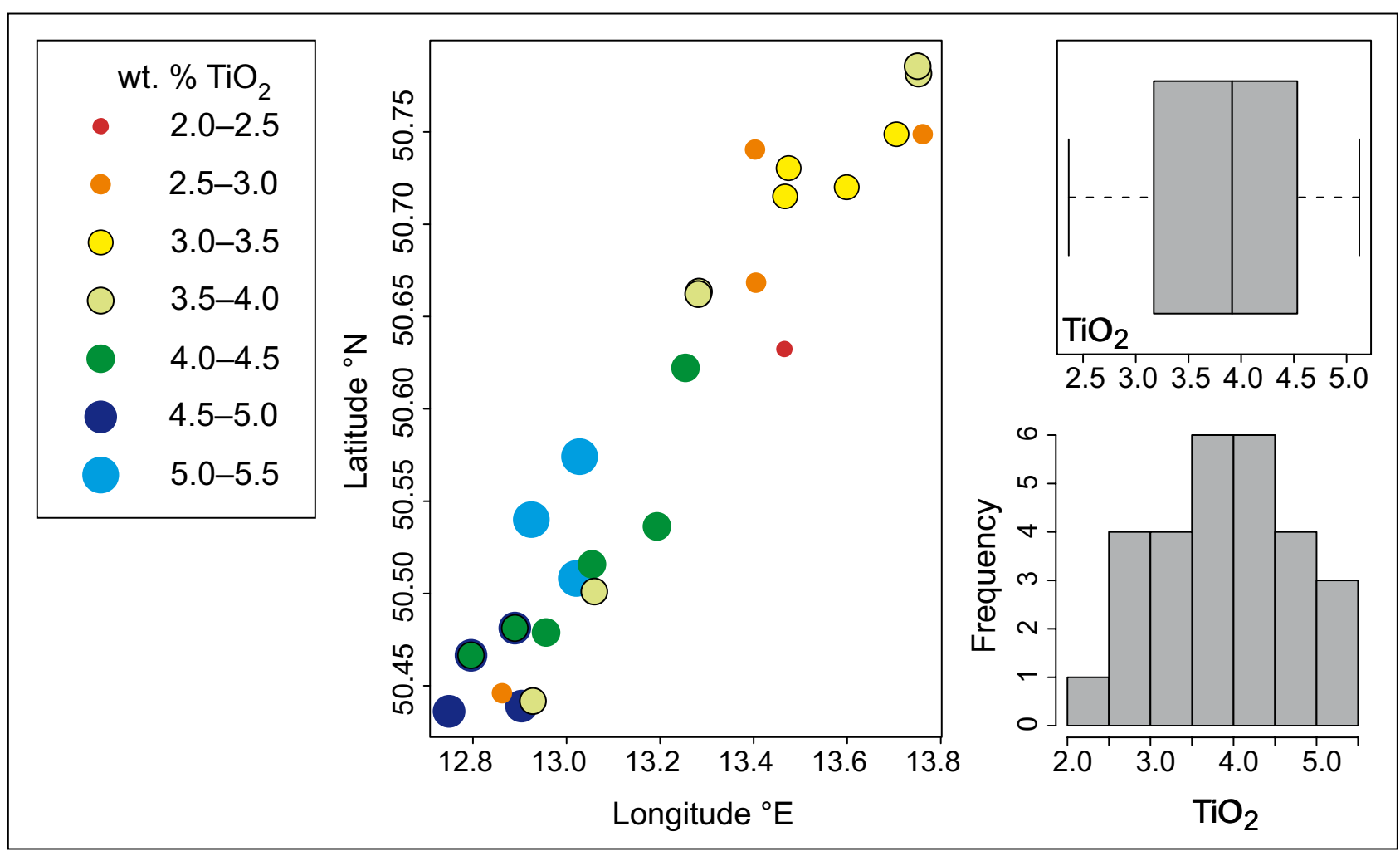

Fig. 4 Regional variation in the $\mathrm{TiO}_{2}$ contents for the studied basaltic rocks.

crystals are subhedral; skeletal forms are rare. Many show signs of deformation. Visible zoning is restricted to the ultimate rim. Characteristic mineral inclusions are magnetite and brown spinel phases; typical alteration products are serpentine minerals and calcite.

\subsubsection{Clinopyroxene}

Generally, seven types of clinopyroxene phenocrysts occur in the studied rocks, of which six are shown in Fig. 5. Every type is characterized by the crystallization of an "outermost overgrowth" (OMG) of beige-brownish colour showing intense internal oscillatory zoning and sector growth features.

Type 1 Clinopyroxene with a light beige core (LBC) of subhedral to anhedral shape. The internal structure of the core shows dissolution features and reaction rims. These cores are always overgrown by the OMG.

Type 2 Similar features like Type 1 and additionally a thin fringe around the anhedral central core characterized by a weaker BSE intensity.

Type 3 Typical green-core clinopyroxenes $(\mathbf{G c C p x})$ as noted by several authors (Huckenholz 1964, 1965, 1966; Duda and Schmincke 1985) with a size between 0.2 and $2 \mathrm{~mm}$. The central green cores are anhedral and show dissolution features and reaction rims; in very rare cases these cores are zoned. The first overgrowth is by a LBCtype pyroxene followed by the OMG-type. The thickness of the respective zones varies.

Type 4 These form the smallest phenocrysts $(0.5 \mathrm{~mm})$ and show internal oscillatory zoning combined with sector zoning features. According to the mineral chemistry (see below) these phenocrysts belong to the OMG-type.

Type 5 Anhedral Cr-rich cores characterize this type as demonstrated by EMPA (see below) and show low BSE intensity $(\mathbf{C r C p x})$. The first overgrowth is by the LBCtype followed by the OMG-generation. It is restricted to lavas from the eastern Erzgebirge.

Type 6 Shows the same features as type 5, but is overgrown merely by the OMG-type.

Type 7 This forms the so called "giant clinopyroxenes" (GCpx), reaching sizes of several centimetres, similar to the LBC-type. These megacrysts are overgrown by a very thin rim of the OMG-type and restricted to the western Erzgebirge.

\subsubsection{Biotite}

Biotite phenocrysts, up to $2 \mathrm{~cm}$ across, occur in some samples from the western Erzgebirge. These have an anhedral, rounded shape and show signs of bending and kinking. Reaction rims formed at the grain boundaries. 


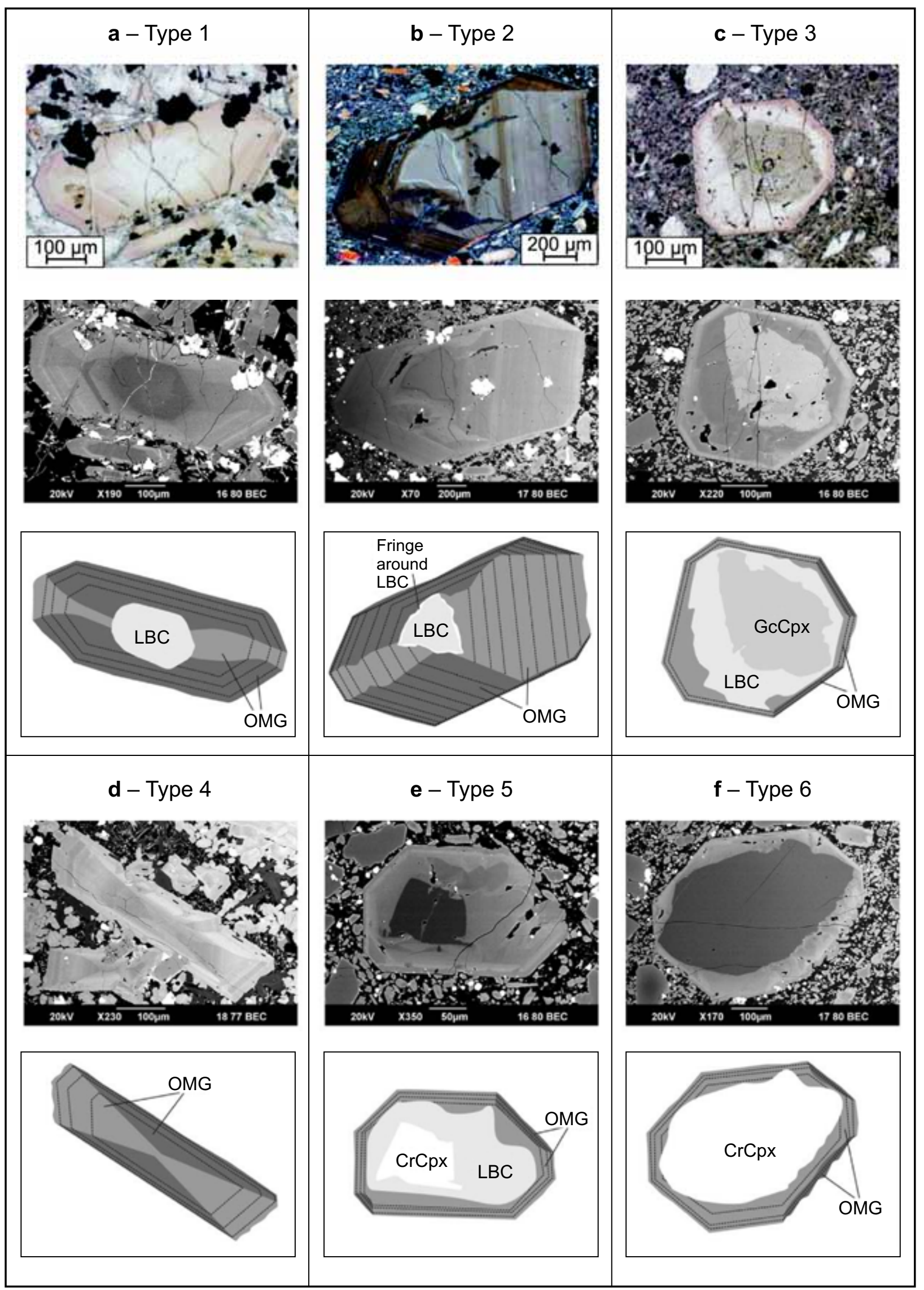




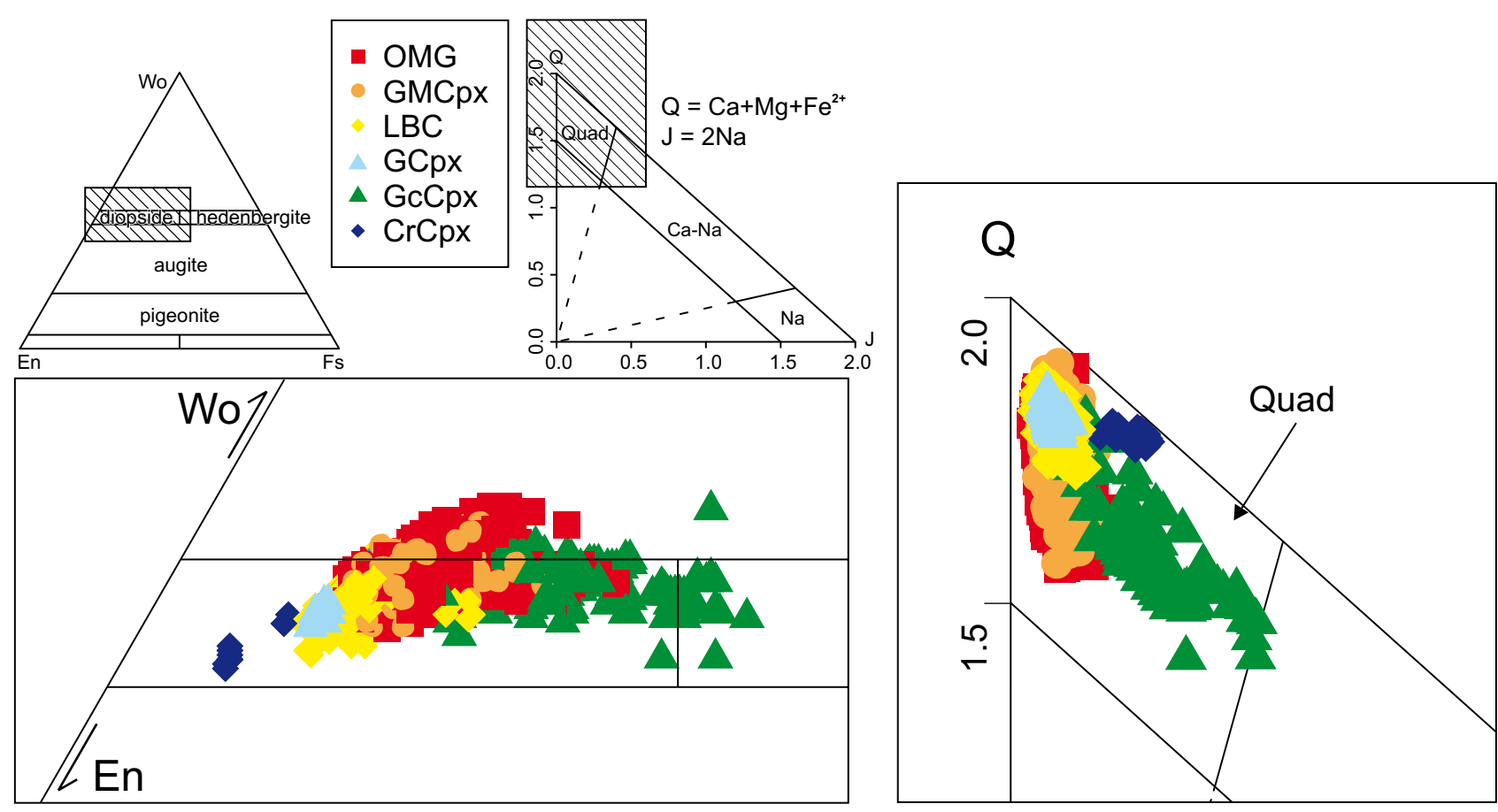

Fig. 6 Plot of analysed clinopyroxenes in classification diagram after Morimoto (1988).

Inclusions of opaque minerals and clinopyroxene are rare. Some of these biotites are intergrown with GCpx.

\subsubsection{Amphibole}

Two samples carry brownish-green subhedral amphibole crystals. These amphiboles are heterogeneous, but show no clear signs of zoning. Typical are inclusions of opaque minerals.

\subsection{Xenoliths and xenocrysts}

Many samples contain xenoliths and xenocrysts of crustal (granitoid, gneiss, and sandstone) and mantle origin (spi-

\section{২}

Fig. 5 Clinopyroxene types in the Erzgebirge basaltic rocks (photomicrographs, back-scattered electron (BSE) images and sketches).

a - Type 1 clinopyroxene $\mathbf{L B C}$ of subhedral to anhedral shape. The internal structure of the core shows dissolution features and reaction rims. The $\mathbf{L B C}$ is overgrown by the OMG-type Cpx; $\mathbf{b}$ - Type 2 resembling the Type 1 but showing additionally a thin fringe around the anhedral LBC core characterized by weaker BSE intensity; $\mathbf{c}-$ Type 3 is a typical green-core clinopyroxene (GcCpx). The green core is anhedral and shows effects of resorption and a reaction rim. The first overgrowth is by a LBC-type followed by the OMG-type clinopyroxene. The thickness of the individual zones varies; $\mathbf{d}$ - Type 4 forms the smallest phenocrysts and shows internal oscillatory zoning combined with sector growth features. These phenocrysts belong exclusively to the OMG-type; $\mathbf{e}$ - Type 5 characterized by anhedral Cr-rich core $(\mathbf{C r C p x})$ with low BSE intensity. The first overgrowth is by the LBC-type followed by the OMG-generation; $\mathbf{f}$ - Type 6 shows the same features like Type 5 but is overgrown merely by the OMG-type. nel lherzolite). The occurrence of mantle-derived material is restricted to samples from the eastern Erzgebirge.

\section{Crystal chemistry of clinopyroxene}

We analyzed 1236 points of the following clinopyroxene types (Tab. 2 and Supp. 4): OMG, groundmass clinopyroxene (GMCpx), GCpx, GcCpx, CrCpx and LBC.

In the conventional IMA classification diagram Morimoto (1988) the compositions of the studied clinopyroxenes plot almost exclusively in the diopside field (Fig. 6) and in the "forbidden" field with Wo $>50$. In detail, the differences between the individual types are shown in the Mg\# versus Si diagram (Fig. 7). The GcCpx have distinctive compositions, richest in iron (some crystals were classified as hedenbergite) and with very low Ti (c. $0.05 \mathrm{apfu})$ and relatively high $\mathrm{Na}(c .0 .11 \mathrm{apfu})$. The CrCpx show the highest $\mathrm{Mg} \#(>87)$ and $\mathrm{Cr}_{2} \mathrm{O}_{3}$ contents (up to 0.8 wt. \%), and the same enriched Na values like the GcCpx. The LBC and GCpx combine relatively high $\mathrm{MgO}$ contents (13-15.1 wt. \%) with comparably low $\mathrm{Al}_{2} \mathrm{O}_{3}$ and $\mathrm{FeO}$ concentrations (c. 5.5 and 5.7 wt. \% respectively). The $\mathrm{TiO}_{2}$ contents are generally lower than those of the OMG and GMCpx (c. 2 wt. \%). Both types show the same crystal chemical features with the highest $\mathrm{TiO}_{2}$ and $\mathrm{Al}_{2} \mathrm{O}_{3}$, accompanied by moderate $\mathrm{MgO}$ and $\mathrm{FeO}$ contents. It has to be stressed that the compositional ranges for individual samples of the given clinopyroxene type may, and often do, overlap significantly with the 


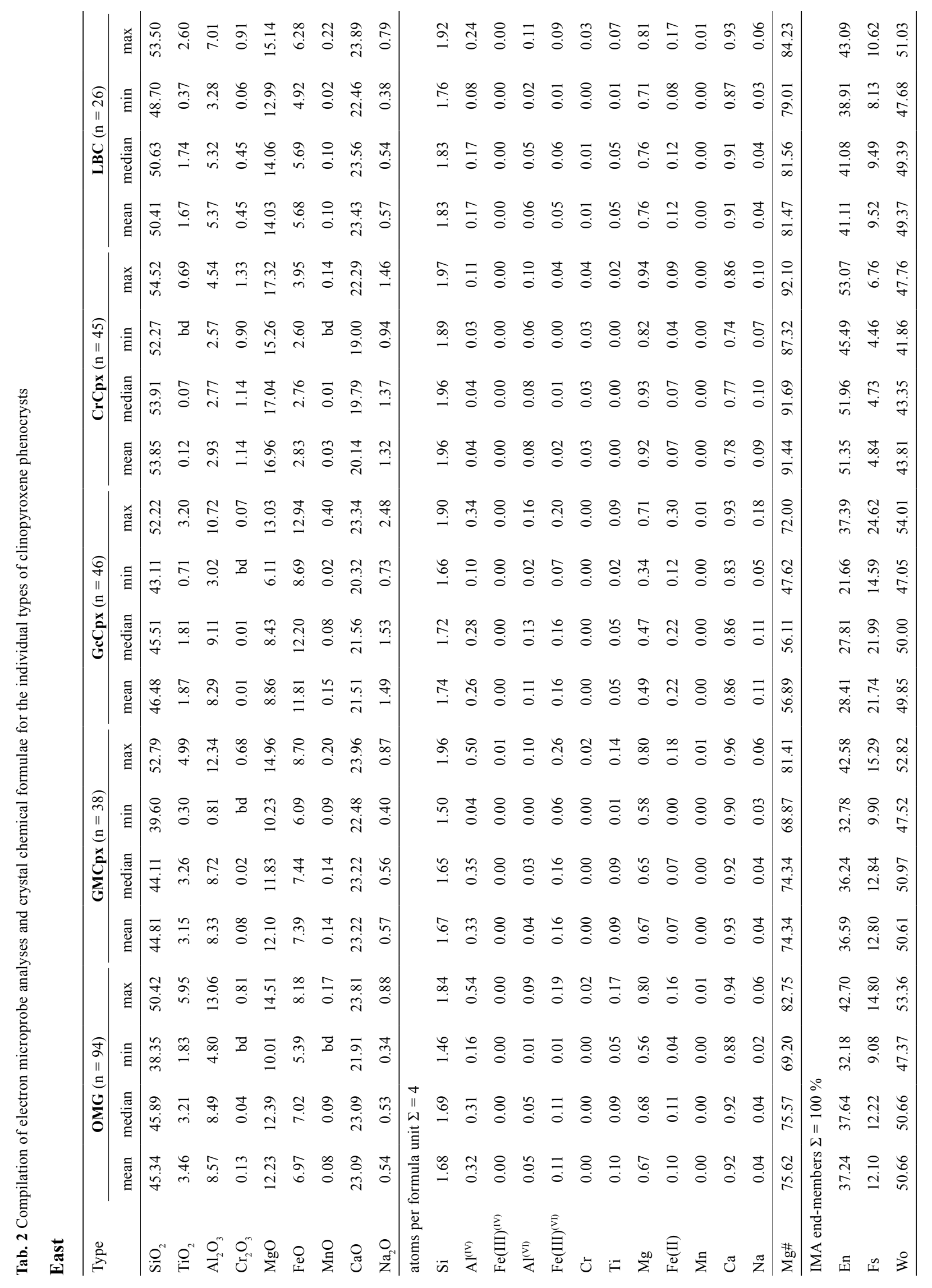




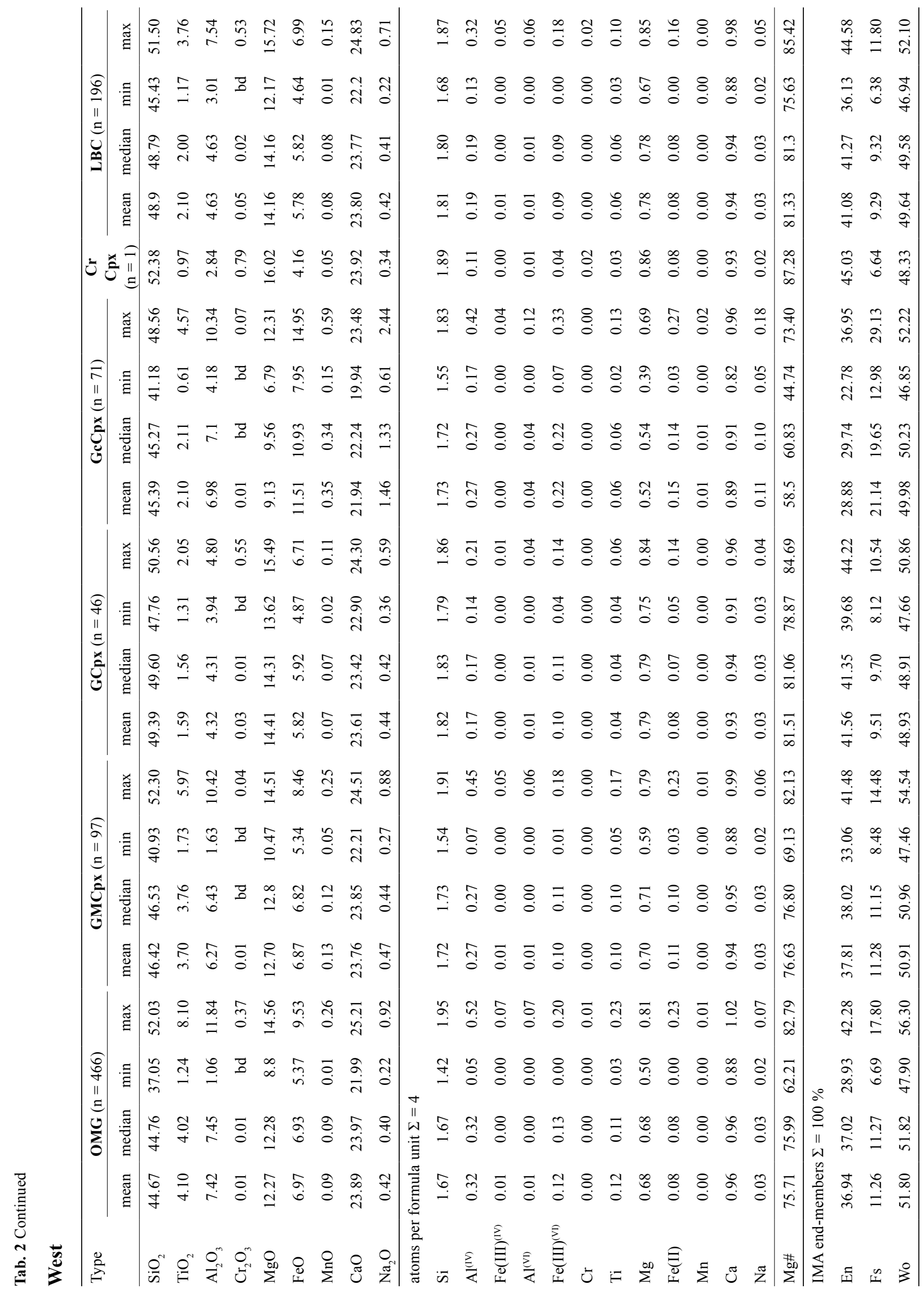




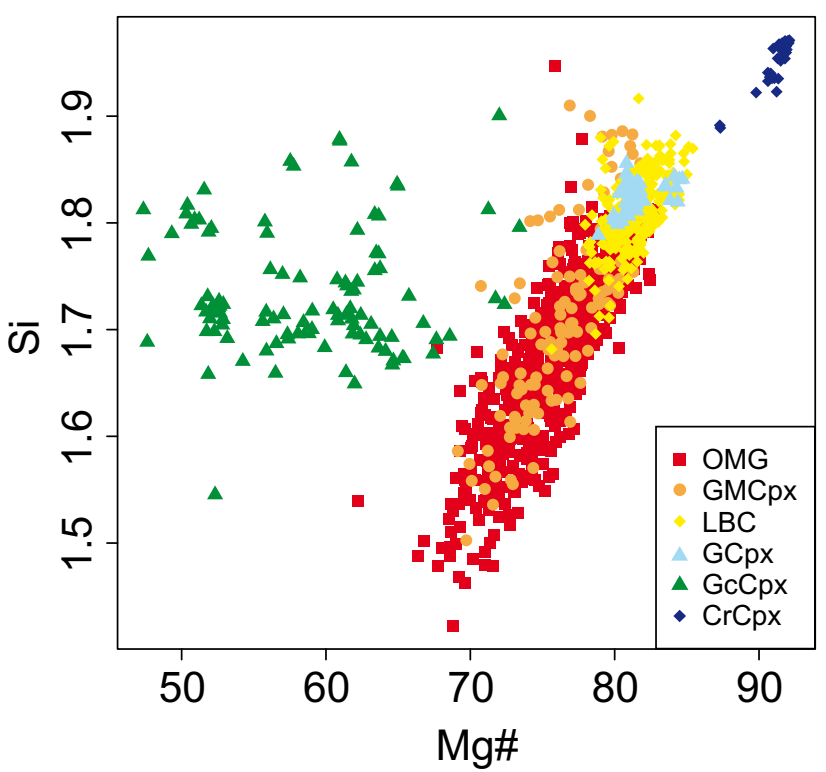

Fig. 7 Binary plot of mg\# vs. Si (formula units) for the six clinopyroxene types.

chemical compositions of the other clinopyroxene types. This is shown, for example, in Fig. 8. All groups have invariably wide range of $\mathrm{Ca}$ contents varying between 0.8 and 0.99 apfu.

The X-ray concentration maps (Supp. 5) provide a good overview of the microstructural and crystal chemical features of the individual clinopyroxene types.

\section{Discussion}

The respective chemical and microstructural data for the clinopyroxene phenocrysts bear a witness of multistage, regionally diverse processes leading to the formation of the Ti-rich as well as Ti-poor basalts in the western and eastern Erzgebirge. It is impossible to constrain rates and mass balances with the data available. In particular, we lack high-resolution age determinations of the lavas as well as trace-element and isotope data for the clinopyroxene. Therefore the following hypothesis remains a tentative one. The presumed origin and evolution of the basaltic rocks in the eastern and western Erzgebirge are summarized in Fig. 9.

The first step was the generation of asthenospheric melts (S1 and $\mathbf{S} 1^{*}$ ) probably from mantle regions with enhanced activities of $\mathrm{H}_{2} \mathrm{O}$ and potassium for the western Erzgebirge, as can be seen from the formation of amphibole and biotite phenocrysts limited to this region.

The chemical characteristics of the $\mathbf{C r C p x}$ are very similar to data from clinopyroxenes of peridotite xenoliths found in some basalts of the eastern Erzgebirge
(A. Renno, unpublished data). Together with xenolithic olivine crystals and mantle-derived peridotite xenoliths, these clinopyroxene crystals were incorporated into the asthenospheric primitive melts $\mathbf{S 1}$ and $\mathbf{S 1}$ * rising rapidly to transient magma chambers or directly to the surface.

The genesis of GcCpx is uncertain with several possibilities: (1) crystallization from a geochemically evolved alkaline melt in the upper mantle (Tappe 2004), (2) nucleation from quartz-bearing magmas in a crustal magma chamber (Bédard et al 1988) or (3) formation at the crust-mantle-boundary (Duda and Schmincke 1985). A multistage process involving crystallization from a melt and subsequent reaction with another melt of contrasting composition seems highly probable. We suggest that these GcCpx were incorporated as xenocrysts into the first magma batches stored in the lithospheric mantle chambers (M1A and M1b). They have reacted with the melts $\mathbf{S} 1$ and $\mathbf{S 1}$ *, whereby they were overgrown by the LBC. Simultaneously, the LBC formed independent, newly grown crystals in these magma chambers. The residence time in the magma chambers associated with the eastern and western parts of the Erzgebirge appears to have been totally different. A short residence demonstrated by the occurrence of mantle-derived xenoliths and xenocrysts in the eastern part (M1a) is in contrast with the western part, where long residence times presumably allowed the settling with complete removal of such xenoliths and xenocrysts as well as the formation of the GCpx in M1b.

The fractionated melts $\mathbf{S 2 a}$ and $\mathbf{S 2 b}$ rose to the shallower magma chambers M2a and M2b, situated probably at a crustal level. These areas appear to be locations of intense magma mixing of the melts $\mathbf{S 2 a}$ or $\mathbf{S 2} \mathbf{b}$ with Ti-rich melts S3a and S3b. The existence of these Ti-rich melts in both parts of the Erzgebirge is demonstrated by the crystallization of the chemically mutually identical OMG and GMCpx phases. The LBC, GCpx, GcCpx, biotite phenocrysts and the mantle-derived olivine xenocrysts all reacted with the newly formed melt. The existence of schlieren-like heterogeneities in the groundmass of some of the lavas suggests that these mixing processes lasted for considerable time and were themselves multistage in character. The geochemical differences among the basaltic rocks demonstrate the significantly lower involvement of the hypothetical Ti-rich melt S3a in these mixing processes in the east.

The ultimate origin of the Ti-rich melts S3a and S3b remains unexplained. Eclogite-derived melts appear as a viable possibility. Melting experiments e.g., by Klemme et al. (2002) showed that the partial melting of eclogite at temperatures similar to, or exceeding $1300{ }^{\circ} \mathrm{C}$ and a pressure of $3 \mathrm{GPa}$ could yield melts enriched in $\mathrm{Ti}$ and Na. Eclogites derived from subducted oceanic crust 

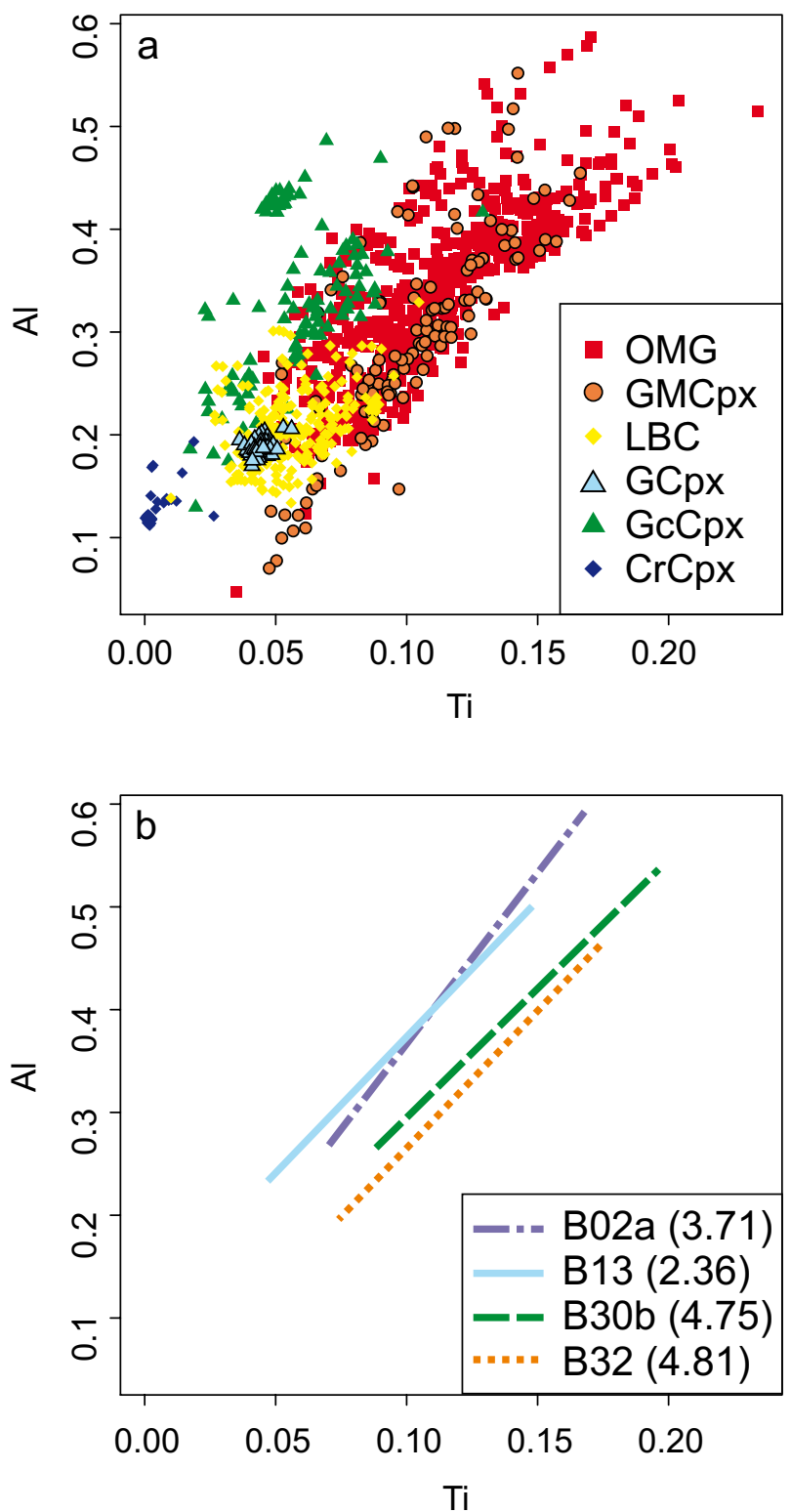

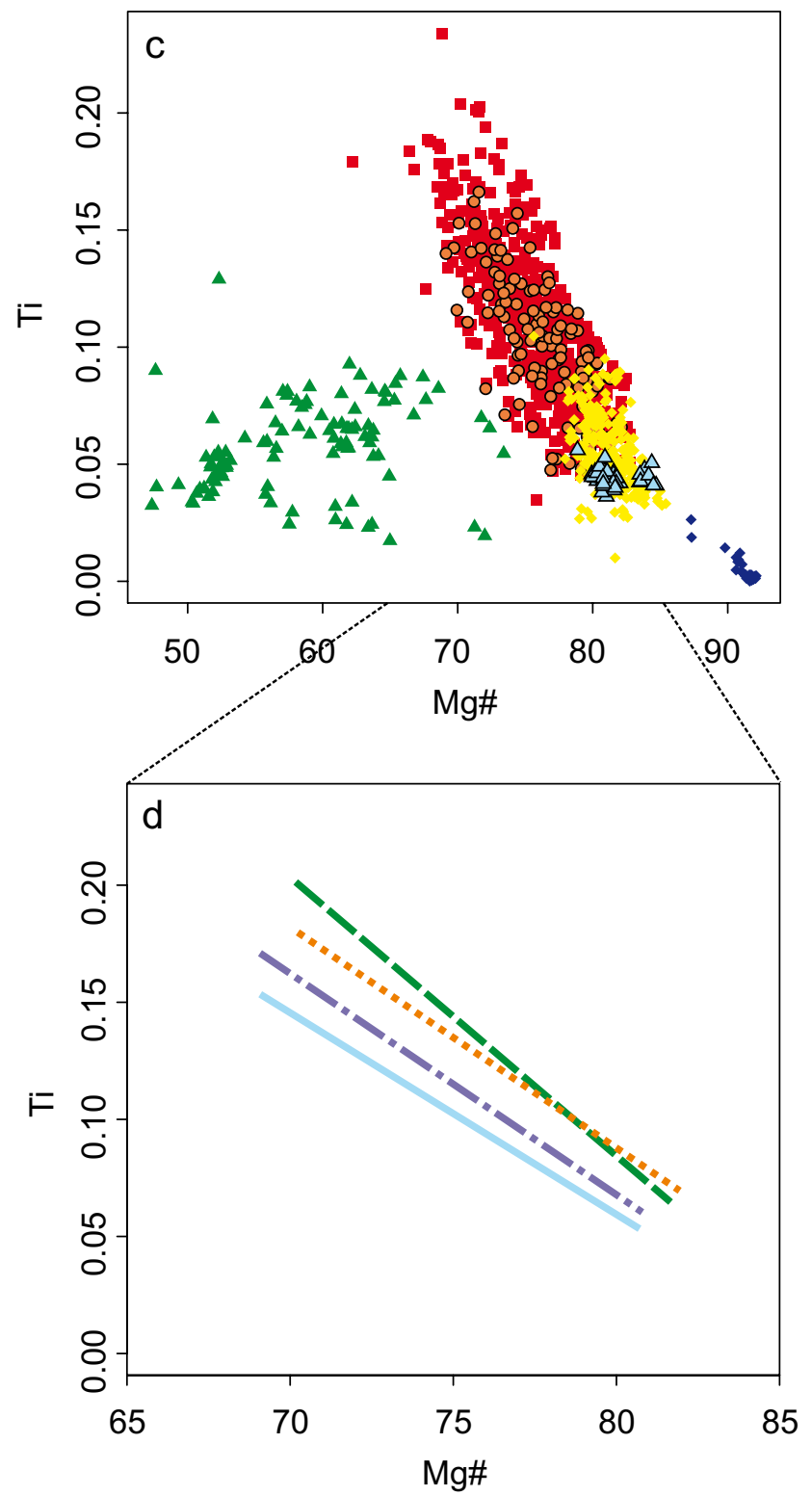

Fig. 8a - Ti vs. Al (formula units) diagram of the individual clinopyroxene types; $\mathbf{b}$ - Ti vs. Al (formula units) diagram showing the variability of data for the OMG-type Cpx using four selected samples as an example (whole-rock $\mathrm{TiO}_{2}$ contents in parentheses); $\mathbf{c}-\mathrm{Mg} \# \mathrm{vs}$. Ti (formula units) diagram of the individual clinopyroxene types; $\mathbf{d}-\mathrm{Mg \#}$ vs. Ti (formula units) diagram showing the range of data for the OMG-type Cpx using four selected samples as an example (whole-rock $\mathrm{TiO}_{2}$ contents in parentheses).

represent an important component of the Saxothuringian lithosphere, as demonstrated by the common presence of exhumed eclogites in the upper crust (e.g., Schmädicke et al. 1992; Konopásek and Schulmann 2005). The mixing of these very hot melts with the $\mathbf{S 1}$ and $\mathbf{S} 2$ magmas in the shallow magma chambers should have triggered the resorption of the GcCpx and the LCB as well as the assimilation of continental crustal material. Indeed, the crustal contamination (biotite flakes and crustal xenoliths) was likely coeval with the mixing in the magma chambers M2a and M2b.

\section{Conclusions}

The synopsis of the petrographic, microstructural and chemical features of several types of clinopyroxenes from cenozoic basaltic rocks in the eastern and the western Erzgebirge demonstrated that the origin of the parental Ti-poor and Ti-rich basaltic melts was a multistage process. At least three different melts were involved in fractionation, assimilation and magma-mixing in two magma chamber systems at different depths, in the lithospheric mantle and in the crust. The regional dichotomy 


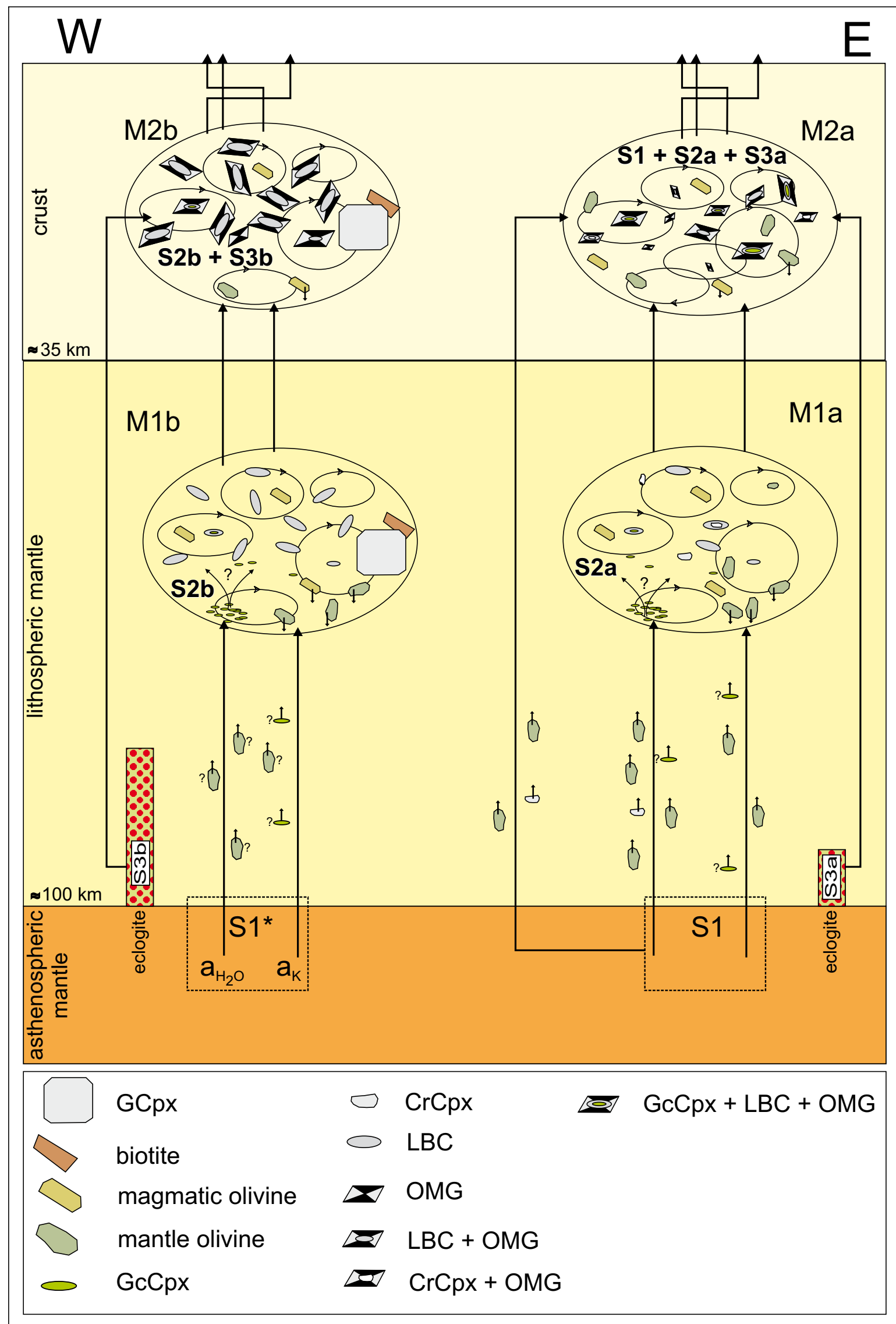


between the eastern and western Erzgebirge basaltic rocks is interpreted by differences in the residence time of the parental magmas in the transient magma chambers, as well as variable mixing with hot Ti-rich melts, possibly derived from an eclogitic source.

Acknowledgements. We gratefully acknowledge the help of A. Pleßow, T. Tschöpe, K. Treptow, and E. Rüdiger with the analytical work. We express our thanks to Jaromír Ulrych and Stanislav Vrána for their careful reviews and to the guest editor Vladislav Rapprich for the subtle and stringent editorial handling of the manuscript. Matthew Huber improved the English style and grammar. This work was funded by the DFG through grant Ha 2568/10-1.

Electronic supplementary material. The GPS coordinates and a map of the sampling sites, detailed petrographic features for the analysed basaltic rocks, the complete set of variation diagrams for the Erzgebirge basaltic rocks including a compilation of the regional

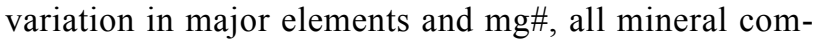
positional data for clinopyroxene and X-ray concentration maps for several typical clinopyroxene crystals are available online at the Journal web site (http://dx.doi. org/10.3190/jgeosci.077).

\section{References}

BABUŠKa V, Plomerová J (1992) The lithosphere in central Europe - seismological and petrological aspects. Tectonophysics 207: 141-163

BABušKa V, Plomerová J (2010) Mantle lithosphere control of crustal tectonics and magmatism of the western Ohře (Eger) Rift. J Geosci 55: 171-186

Babuška V, Plomerová J, Fischer T (2007) Intraplate seismicity in the western Bohemian Massif (central Europe): a possible correlation with a paleoplate junction. J Geodyn 44: 149-159

BÉDARD JH, Francis DM, LudDEN J (1988) Petrology and pyroxene chemistry of Monteregian dykes; the origin of concentric zoning and green cores in clinopyroxenes from alkali basalts and lamprophyres. Can J Earth Sci 25: 2041-2058

Fig. 9 Tentative model for the formation of the basaltic rocks in the western (W, labelled by 'a') and eastern (E, labelled by 'b') Erzgebirge, respectively. $\mathbf{S 1}=$ primary asthenospheric melt (E Erzgebirge); $\mathbf{S 1}$ * = primary asthenospheric melt with higher $\mathrm{a}_{\mathrm{H} 2 \mathrm{O}}$ and $\mathrm{a}_{\mathrm{K}}$ (W Erzgebirge); S2a, $\mathbf{S 2} \mathbf{b}=$ fractionated melts from the first magma chamber system in the lithospheric mantle; S3a, S3b = eclogite-derived melts; M1a, M1b = first magma chamber in the lithospheric mantle; M2a, M2b $=$ second (crustal) magma chamber systems.
BogaARd PJF, WÖRner G (2003) Petrogenesis of basanitic to tholeiitic volcanic rocks from the Miocene Vogelsberg, Central Germany. J Petrol 44: 569-602

Duda A, Schmincke H-U (1985) Polybaric differentiation of alkali basaltic magmas: evidence from green-core clinopyroxenes (Eifel, FRG). Contrib Mineral Petrol 91: 340-353

Duncan AR (1987) The Karoo Igneous Province - a problem area for inferring tectonic setting from basalt geochemistry. J Volcanol Geotherm Res 32: 13-34

Dupuy C, Marsh J, Dostal J, Michard A, Testa S (1988) Asthenospheric and lithospheric sources for Mesozoic dolerites from Liberia (Africa): trace element and isotopic evidence. Earth Planet Sci Lett 87: 100-110

Essawy MA, El-Metwally AA (1999) Petrogenesis of a high $\mathrm{TiO}_{2}$ mafic dyke swarm from southwest Sinai. J Afr Earth Sci 29: 551-565

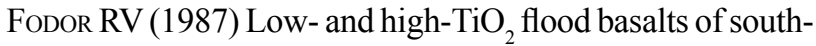
ern Brazil: origin from picritic parentage and a common mantle source. Earth Planet Sci Lett 84: 423-430

Gibson SA, Thompson RN, Dickin AP, Leonardos OH (1995) High-Ti and low-Ti mafic potassic magmas: Key to plume-lithosphere interactions and continental flood-basalt genesis. Earth Planet Sci Lett 136: $149-165$

Goes S, Loohuis JJP, Wortel MJR, Govers R (2000) The effect of plate stresses and shallow mantle temperatures on tectonics of northwestern Europe. Global Planet Change 27: 23-38

HAASE KM, RenNo AD (2008) Variation of magma generation and mantle sources during continental rifting observed in Cenozoic lavas from the Eger Rift, Central Europe. Chem Geol 257: 192-202

Hrubcová P, Środa P, Špičák A, Guterch A, Grad M, Keller GR, Brueckl E, Thybo H (2005) Crustal and uppermost mantle structure of the Bohemian Massif based on CELEBRATION 2000 data. J Geophys Res B Solid Earth 110: 1-21, DOI: 10.1029/2004JB003080

HuckenHolz HG (1964) Der petrogenetische Werdegang der Klinopyroxene in den tertiären Vulkaniten der Hocheifel I. Die Klinopyroxene der Alkaliolivinbasalt-Trachyt-Assoziation. Contrib Mineral Petrol 11: 138-195

HuCKENHOLz HG (1965) Der petrogenetische Werdegang der Klinopyroxene in den tertiären Vulkaniten der Hocheifel II. Die Klinopyroxene der Basanitoide. Contrib Mineral Petrol 11: 415-448

HuCKenHOlz HG (1966) Der petrogenetische Werdegang der Klinopyroxene in den tertiären Vulkaniten der Hocheifel. Contrib Mineral Petrol 12: 73-95

Jama Aden A, Frizzo P (1996) Geochemistry and origin of low and high $\mathrm{TiO}_{2}$ mafic rocks in the Barkasan Complex: a comparison with common Neoproterozoic gabbros of northern Somali crystalline basement. J Afr Earth Sci 22: $43-54$ 
JANOUŠEK V, FARROW CM, ERBAN V (2006) Interpretation of whole-rock geochemical data in igneous geochemistry: introducing Geochemical Data Toolkit (GCDkit). J Petrol 47: 1255-1259

Jung S, Hoernes S (2000) The major- and trace-element and isotopic ( $\mathrm{Sr}, \mathrm{Nd}, \mathrm{O})$ geochemistry of Cenozoic alkaline rift-type volcanic rock from the Rhön area (central Germany): petrology, mantle source characteristics and implications for asthenosphere-lithosphere interactions. J Volcanol Geotherm Res 99: 27-53

Kaiser G, Pilot J (1986) Weitere K-Ar-Datierungen an jungen Vulkaniten. Z geol Wiss 14: 121-124

Katzir Y, Litvinovsky B, Eyal M, Zanvilevich A, VAPNiK Y (2006) Four successive episodes of Late Pan-African dikes in the central Elat area, southern Israel. Isr J Earth Sci 55: 69-93

Klemme S, Blundy JD, Wood BJ (2002) Experimental constraints on major and trace element partitioning during partial melting of eclogite. Geochim Cosmochim Acta 66: 3109-3123

Konopásek J, Schulmann K (2005) Contrasting Early Carboniferous field geotherms: evidence for accretion of a thickened orogenic root and subducted Saxothuringian crust (Central European Variscides). J Geol Soc, London 162: $463-470$

Le BAs MJ (1989) Nephelinitic and basanitic rocks. J Petrol 30: 1299-1312

Le Bas MJ, Le Maitre RW, Streckeisen A, Zanettin B, IUGS Subcomission on the Systematics of Igneous Rocks (1986) A chemical classification of volcanic rocks based on the total alkali-silica diagram. J Petrol 27: 745-750

Le Maitre RW, Streckeisen A, Zanettin B, Le Bas MJ, Bonin B, BAteman P (2005) Igneous Rocks: A Classification and Glossary of Terms: Recommendations of the International Union of Geological Sciences Subcommission on the Systematics of Igneous Rocks. Cambridge University Press, Cambridge, pp 1-252

MaLkovský M (1987) The Mesozoic and Tertiary basins of the Bohemian Massif and their evolution. Tectonophysics 137: 31-42

Middlemost EAK (1994) Naming materials in the magma/ igneous rock system. Earth Sci Rev 37: 215-224

Mlčoch B, Konopásek J (2010) Pre-Late Carboniferous geology along the contact of the Saxothuringian and Teplá-Barrandian zones in the area covered by younger sediments and volcanics (western Bohemian Massif, Czech Republic). J Geosci 55: 81-94

Morimoto N (1988) Nomenclature of pyroxenes. Bull Minéral 111: 535-550

Murphy JB (1988) Late Precambrian to Late Devonian mafic magmatism in the Antigonish Highlands of Nova Scotia: multistage melting of a hydrated mantle. Can J Earth Sci 25: 473-485
Niese S, Pfeiffer L, Gleisberg GB (1995) Geochemie sächsischer Tertiärmagmatite. Z geol Wiss 23: 317-330

PAPIKE JJ, VAniman DT (1978) The Lunar Mare Basalt suite. Geophys Res Lett 5: 433-436

Papike JJ, Hodges FN, Bence AE, Cameron M, Rhodes JM (1976) Mare basalts: crystal chemistry, mineralogy, and petrology. Rev Geophys 14: 475-540

Pfeiffer L (1978) Beitrag zur Petrochemie der sächsischen Tertiärvulkanite. Freiberg Forsch H C333: 1-164

Pik R, Deniel C, Coulon C, Yirgu G, Hofmann C, Ayalew D (1998) The northwestern Ethiopian Plateau flood basalts: classification and spatial distribution of magma types. J Volcanol Geotherm Res 81: 91-111

Pik R, Marty B, Hilton DR (2006) How many mantle plumes in Africa? The geochemical point of view. Chem Geol 226: 100-114

Pilot J, Pfeiffer L, Rösler HJ, Schlichting M, Kaiser G (1984) Zur genetischen Problematik der tertiären Vulkanite von der Lausitz und des Erzgebirges auf Grund von Strontiumisotopenverhältnissen, $\mathrm{Rb}-\mathrm{Sr}$ - und $\mathrm{K}-\mathrm{Ar}$ Altern. Freiberg Forsch H C389: 84-92

Plomerová J, Achauer U, Babuška V, Vecsey L (2007) Upper mantle beneath the Eger Rift (Central Europe): plume or asthenosphere upwelling? Geophys J Int 169: 675-682

Prodehl C, Müller S, HaAk V (1995) The European Cenozoic rift system. In: OLSEN KH (ed) Continental Rifts: Evolution, Structure, Tectonics. Elsevier, Amsterdam, pp 133-212

RAPPRICH V (2005) Compositional variation of clinopyroxenes of basaltic, essexitic and tephriphonolitic rocks from the Doupovské hory Volcanic Complex, NW Bohemia. J Czech Geol Soc 50: 119-132

RöNICK R (2010) Geochemisch-petrologische Untersuchungen an Ti-reichen Basalten des Erzgebirges. Unpublished Diploma thesis, TU Bergakademie Freiberg - Institut für Geologie, pp 1-124

Schmädicke E, Okrusch M, Schmidt W (1992) Eclogitefacies rocks in the Saxonian Erzgebirge, Germany: high pressure metamorphism under contrasting $\mathrm{P}-\mathrm{T}$ conditions. Contrib Mineral Petrol 110: 226-241

SHRBENÝ O (1995) Chemical composition of young volcanites of the Czech Republic. Czech Geol Survey Spec Pap 4: 1-53

TAPPE S (2004) Mesozoic mafic alkaline magmatism of southern Scandinavia. Contrib Mineral Petrol 148: 312-334

TodT W, Lippolt HJ (1975) K-Ar Altersbestimmungen an Vulkaniten bekannter paläomagnetischer Feldrichtung II Sachsen. J Geophysics 41: 641-650

UlRYCH J (1986) Clinopyroxenes in the Cenozoic volcanics of the České středohoří Mts.: a review. Acta Univ Carol, Geol 1986: 117-131

Ulrych J, Pivec E, Rutšek J, Povondra P (1990) Olivines-monticellites and clinopyroxenes in melilitic rocks, 
Ploučnice River region, Czechoslovakia. Acta Univ Carol, Geol 1990: 141-164

Ulrych J, Svobodová J, Balogh K (2002) The source of Cenozoic volcanism in the České středohoří Mts., Bohemian Massif. Neu Jb Mineral, Abh 177: 133-162
Ulrych J, Lloyd Fe, Balogh K, Hegner E, Langrová A, Lang M, Novák JK, ŘAnda Z (2005) Petrogenesis of alkali pyroxenite and ijolite xenoliths from the Tertiary Loučná-Oberwiesenthal Volcanic Centre, Bohemian Massif in the light of new mineralogical, geochemical, and isotopic data. Neu Jb Mineral, Abh 182: 57-79 
